\title{
The Diversity of Hornets in the Genus Vespa (Hymenoptera: Vespidae; Vespinae), Their Importance and Interceptions in the United States
}

\author{
Allan H. Smith-Pardo, ${ }^{1,4}$ James M. Carpenter, ${ }^{2}$ and Lynn Kimsey ${ }^{3}$ \\ 'USDA-APHIS-PPQ, Science and Technology (S\&T), Sacramento, CA, ${ }^{2}$ Department of Invertebrate Zoology, American Museum \\ of Natural History, New York, NY, ${ }^{3}$ Bohart Museum of Entomology, University of California, Davis, Davis, CA, and ${ }^{4}$ Corresponding \\ author, e-mail: allan.h.smith-pardo@usda.gov
}

Subject Editor: Heather Hines

Received 20 December, 2019; Editorial decision 11 March, 2020

\begin{abstract}
Hornets in the genus Vespa (Vespidae, Vespinae) are social wasps. They are primarily predators of other insects, and some species are known to attack and feed on honeybees (Apis mellifera L.), which makes them a serious threat to apiculture. Hornet species identification can be sometimes difficult because of the amount of intraspecific color and size variation. This has resulted in many species-level synonyms, scattered literature, and taxonomic keys only useful for local populations. We present a key to the world species, information on each species, as well as those intercepted at United States Ports of Entry during the last decade. Images of all the species and some of the subspecies previously described are also included.
\end{abstract}

\section{Resumen}

Los avispones (Vespidae: Vespinae: Vespa) son avispas sociales, depredadoras de otros insectos y algunas de las especies muestran cierta preferencia por abejas, incluyendo las abejas melíferas (Apis mellifera L.) convirtiéndose en una amenaza para la apicultura. La identificación de las especies puede ser difícil dado que muchas especies muestran una gran variación inter-especifica que ha tenido como resultado multitud de sinonimias en el género y además, porque la literatura en el género es dispersa y las claves taxonómicas solo funcionan para poblaciones locales. En este trabajo, presentamos una clave para las especies del mundo, información para cada especie así como de las especies interceptadas en puertos de entrada a los Estados Unidos durante la última década. Se incluyen además imágenes para todas las especies así como para algunas de las subespecies previamente descritas.

Key words: invasive species, quarantine significance, pest, diversity, social wasp

Hornets in the genus Vespa are large, predatory, eusocial wasps native to Europe and Asia. They prey on a wide diversity of insects, but several species are predatory on honeybees. Vespa nests can be physically large, with over 1,000 workers, but usually with hundreds of workers (Archer 2008). Nests can be aerial, attached to tree branches or in shrubs, in crevices, under eaves or underground depending on the species. Depending on the latitude, nests can be either annual, started by a new queen every spring, or perennial, where young queens take over from old ones (Matsuura and Yamane 1990). Colonies in warm tropical climates tend to be perennial.

These wasps exhibit a range of color patterns that vary geographically. Previous authors have named numerous subspecies based solely on color patterns, but Vespa color patterns tend to grade from one region to the next Nguyen et al. (2006), Perrard et al. (2014). These subspecies were all synonymized by Carpenter and Kojima (1997).

Beggs et al. (2011) assessed the distribution, abundance, impact, and management of invasive species of Vespidae worldwide. In the case of the hornets, several Vespa species have been introduced outside their native ranges, including V. crabro Linnaeus, 1758 (Hymenoptera: Vespidae) from Europe into the United States (Bequaert 1932, Shaw and Weidhaas 1956); V. velutina Lepeletier, 1836 (Hymenoptera: Vespidae) from Asia into Europe (Monceau et al. 2014); and V. tropica Linnaeus, 1758 (Hymenoptera: Vespidae) from continental Asia into the island of Guam (Anonymous 2016). There are also records of other species collected outside their ranges without becoming established (see Kimsey and Carpenter 2012 for North America). 
Some of the introduced Vespa species have shown to be environmental stressors as predators of already declining populations of native insects, spiders, and honeybees (Shah and Shah 1991, Abrol 1994, Choi et al. 2011, among others). They can also be competitors for food and nesting sites of native wasps (Cini et al. 2018), vectors of parasites or diseases that can affect honey bees and native wasps (Choi et al. 2011), and impact human safety because of their aggressiveness and fatal reactions to their venom (Nguyen et al. 2010, Kularatne et al. 2014).

The invasion of $V$. velutina into Europe typifies these problems. These hornets prey on the domestic honey bee [Apis mellifera (Linnaeus) (Hymenoptera: Apidae)], disrupt the ecological role of honeybees as pollinators (Villemant et al. 2006a, b; Monceau et al. 2014), have potentially altered local biodiversity (Fedele et al. 2019), and are potentially deadly to people allergic to their stings.

In Europe, the native hornet, V. crabro, is protected in some regions, such as in Germany (Federal Species Protection OrdinanceBArtSchV/Federal Nature Conservation Act-BNatSchG). The introduced $V$. velutina may compete for food resources with V. crabro, which is a more generalist predator (Shaw and Weidhaas 1956, Baracchi et al. 2010). According to Monceau et al. (2014), some beekeepers have reported increased $V$. crabro predation on honeybees since the introduction of V. velutina in Europe.

This species was introduced into New York State in the mid1800s (de Saussure 1898). Since then, it has since spread throughout eastern North America (Kimsey \& Carpenter 2012). Vespa tropica was first found on Guam in 2016 (Anonymous 2016). Since then, it has become so widespread on the island it is no longer under quarantine, even though problems have arisen because it is aggressive and may nest close to human settlements.

Studies by Blanchard et al. (2008) and Yañez et al. (2012) had shown the potential transmission of the Israeli Acute Paralysis Virus (IAPV), one of the causes of Colony Collapse Disorder in honeybees (Cox-Foster et al. 2007) between honeybees and their non-Apis predators, such as hornets. The possibility of transmission from hornets to honeybees or other native species has yet to be determined.

The introduction of alien hornet species into the United States is a real risk and having a practical synthesis of the current knowledge on the basic biology and identification of the world's hornets would greatly increase our capacity to identify and enhance the detection of potentially invasive species of hornets or to monitor their dispersion if they are introduced.

In this study, we provide information on the species diversity of hornets, taxonomic keys and visual aids for the identification of the different species.

\section{Materials and Methods}

We performed an Ad hoc/ unlimited search for interceptions of species of Vespinae (Vespa and Vespula Thomson) at Ports of Entry (PE) to the United States using the USDA-APHIS, Plant Protection and Quarantine (PPQ) databases AQAS (Agricultural Quarantine Activity Systems) and ARM (Agricultural Risk Management System) going back to 2010. After the search, the data were processed, filtered and the total of interceptions for hornets (genus Vespa) was determined for the years 2010 to 2018. In addition, some information from interception records, such as origin and means of transportation, is given in the results and the discussion.

Taxonomic keys provided below were constructed in part based on previous keys by Archer (1989, 2012). A revised, fully illustrated key was needed to clarify and simplify diagnostic characters used in previous keys. In addition to the keys, we provide, for the first time, high-resolution images of these features.
Specimens used for the images were from the American Museum of Natural History (AMNH), New York, NY (C. Lebeau) and from the Bohart Museum of Entomology (BME), University of California, Davis (S. Heydon). Images were captured and edited using a Nikon SMZ18 System with a Nikon DS-fi2 camera, the stacking software, Helicon Focus 6.0, and Photoshop Elements 12.

Typerepositoriesaregivenin parenthesesat theend of speciesentries in synonym lists. These include BASEL = Naturhistorisches Museum, Basel, Switzerland; BERLIN = Museum für Naturkunde, Berlin, Germany; BUDAPEST = Zoological Department, Hungarian Natural History Museum, Budapest, Hungary; CALCUTTA = Zoological Survey of India, Calcutta; CAMBRIDGE = Museum of Comparative Zoology, Harvard University, Cambridge, Massachusetts, USA; COPEHAGEN = Statens Naturhistoriske Museum, Copenhagen, Denmark; FUKUOKA = Kitakyushu Museum of Natural History and Human History, Fukuoka, Japan; GENOA = Museo Civico di Storia Naturale di Genova, Genoa, Italy; KAGOSHIMA = Reimeikan-Kagoshima History Museum, Kagoshima, Japan; KUNMING = Kunming Natural History Museum of Zoology, Kunming, Yunnan, China; LEIDEN = Rijksmuseum van Natuurlijke Historie (now called the Nederlands Centrum voor Biodiversiteit Naturalis), Leiden, Netherlands; LOGAN = The American Entomological Institute, Logan, Utah, USA; LONDON-LS = Linnaean Society, London, England; LONDON-NHM = The Natural History Museum, London, England; MUNICH = Zoologische Staatssammlung München, Munich, Germany; OXFORD = Hope Entomological Collections, Oxford University Museum, Oxford, England; PARIS = Museum National d'Histoire Naturelle, Laboratoire d'Entomologie, Paris, France; SAPPORO = Entomological Institute, Hokkaido University, Sapporo, Japan; ST. PETERSBURG = Zoological Museum of the Zoological Institute, Saint Petersburg, Russia; TAICHUNG = Taiwan Agricultural Research Institute, Taichung, Taiwan; TOKYO = National Museum of Nature and Science, Tokyo, Japan; TURIN = Museo Regionale di Scienze Naturali, Turin, Italy; UPPSALA = Uppsala universitet Evolutionsmuseet, Uppsala, Sweden; VENICE = Museo di Storia Naturale di Venezia Venice, Italy; VIENNA = Naturhistorisches Museum, Vienna, Austria; WASHINGTON = Smithsonian National Museum of Natural History, Washington, DC, United States.

\section{Genus Vespa Linnaeus}

Vespa Linnaeus, 1758: 343. Type species: 'Vespa crabro Fab'. (= Vespa crabro Linnaeus, 1758); designated by Latreille, 1810: 438. Macrovespa Dalla Torre, 1904: 64 (subgenus of Vespa Linnaeus). Type species Vespa crabro Linnaeus, 1758. Designated by Bequaert, 1930: 64.

Nyctovespa van der Vecht, 1959: 210 (subgenus of Vespa Linnaeus). Type species: Vespa binghami du Buysson, 1905, original designation.

Diagnosis. The subfamily Vespinae can be distinguished from other Vespidae by the hind wings lacking an anal lobe (Fig. 1a); forewing recurrent veins ending in the same submarginal cell (Fig. 1b), marginal cell narrowly pointed along costal vein (not extending away from it) (Fig. 1b); pronotal lobe separated from tegula by a distance equal or less than its length (Fig. 1c); tarsal claws simple, not bifid (Fig. 1d), without parategula (Fig. 1e), and metasoma sessile with first tergum truncate (Fig.1f).

Species of the genus Vespa can be distinguished from other vespine genera by having both the pronotal and the pretegular carinae 


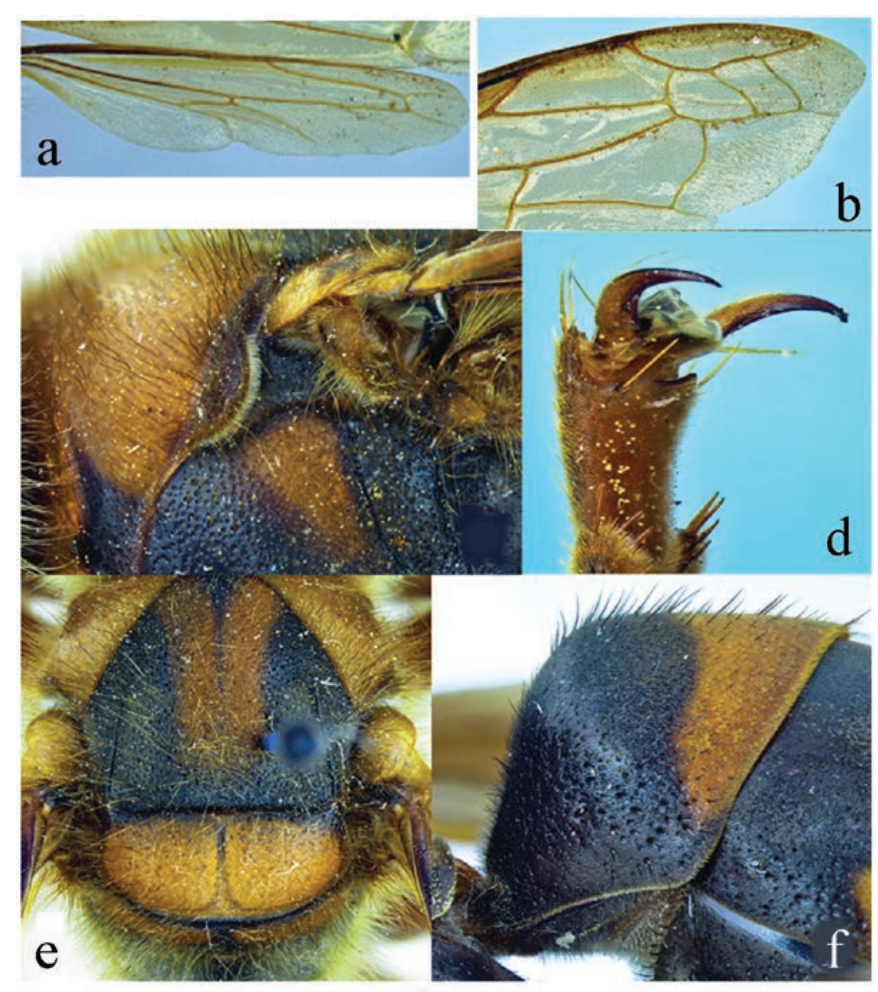

1.
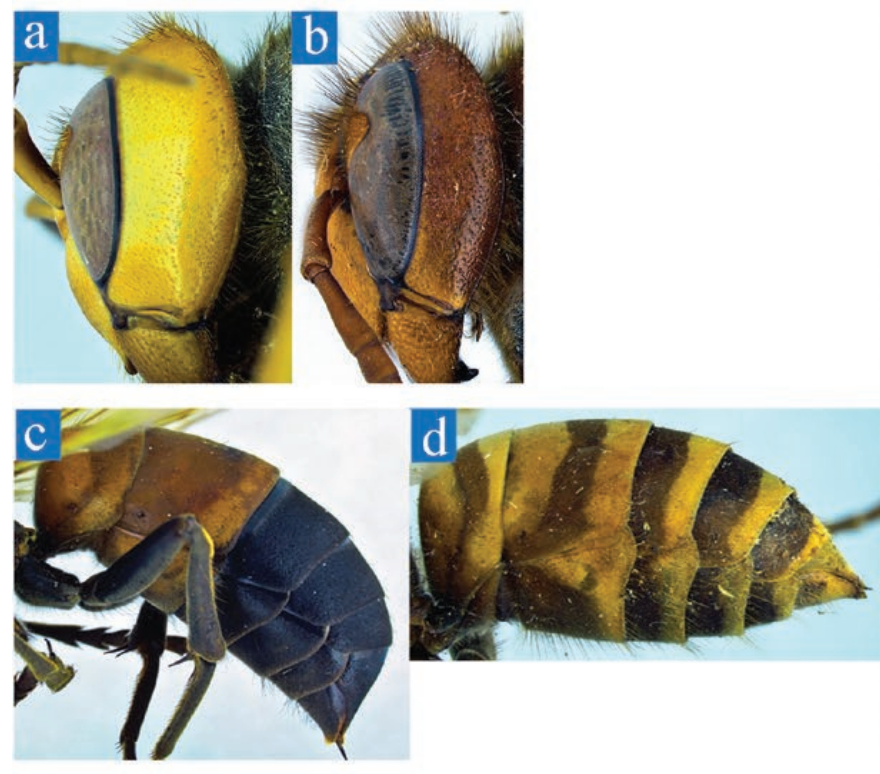

3.
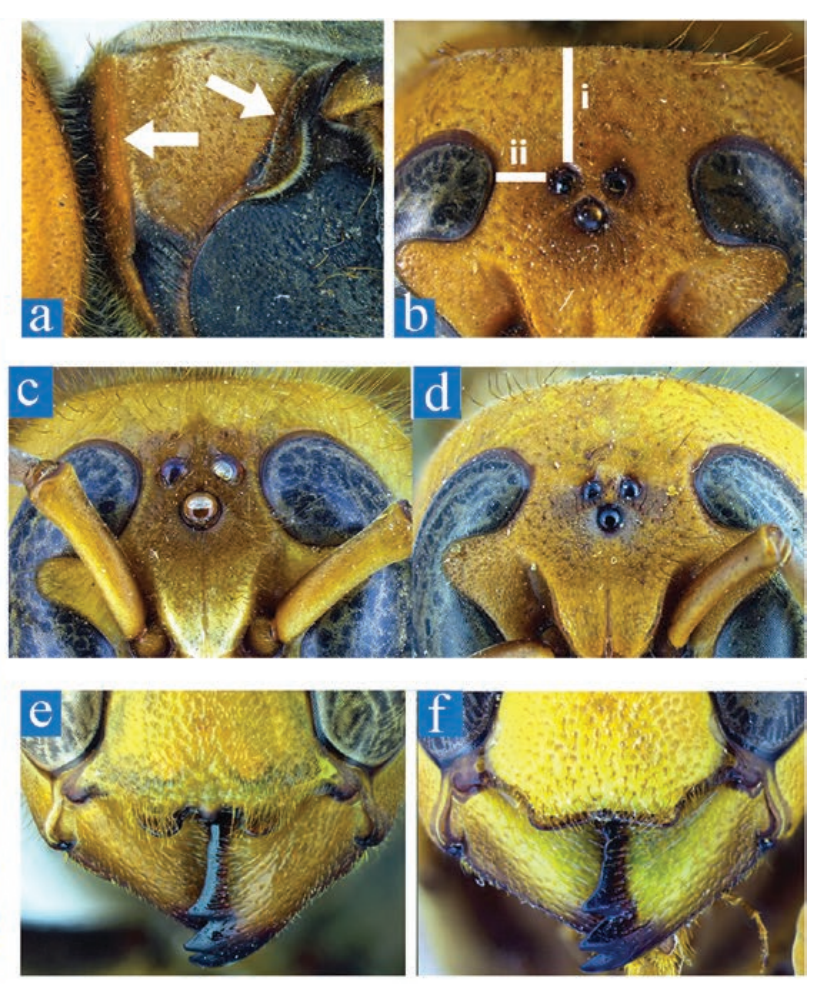

2.
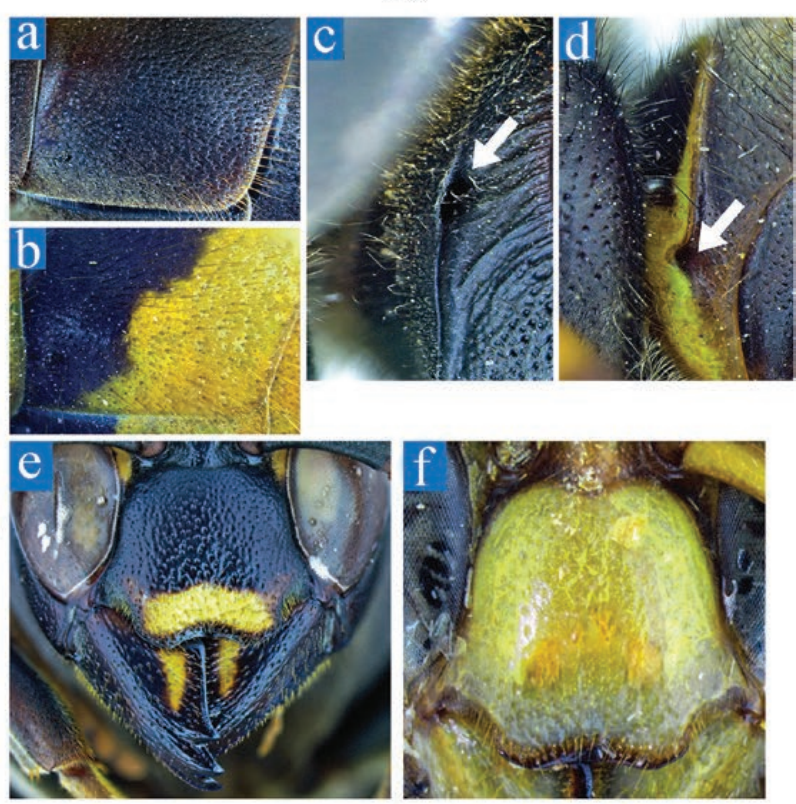

4.

Figs. 1-4. Vespa morphology. Fig. 1 (a) Hindwing. (b) Forewing apex. (c) Lateral view of pronotal lobe. (e) Hindtarsal claws. (f) Thoracic dorsum. (g) Lateral view of metasomal segment 1. Fig. 2. (a) Lateral view of pronotal lobe. (b) Dorsal view of vertex, i=distance from hind ocellus to posterior head margin, ii=distance between lateral ocellus and eye. (c) Lateral view of metasomal segment 1. (d) Costal margin of forewing apex. (e) Dorsal view of vertex. (c) Front view of clypeus and mandibles. Fig. 3. (a, b) Lateral view of head. (c, d) Lateral view of metasoma. (3a, 3c) V. soror. Fig. 4. (a, b) Lateral view of metasomal tergum 2. (c, d) Lateral view of pronotal carina. (e, f) Front view of clypeus. Figs. 1a-g, 2a, 2b, 3b, 3d, V. crabro. Fig. 2c, V. binghami. Figs. 2d, 2e, V. analis. Fig. 2f, V. bicolor. Figs. 4a, 4e, V. fervida. Fig. 4b, V. multimaculata. Figs. 4c, 4f, V. luctuosa. Fig. 4d, V. affinis.

(Fig. 2a), head vertex with the distance from the posterior ocellus to the posterior margin of vertex more than twice the distance between the posterior ocelli and the compound eye (Fig. 2b), the basal metasomal segment is anteriorly rounded (Fig. 1f), and the length of the forewing prestigma is three times or more the length of the pterostigma (Fig. 1b).

\section{Keys to the Queens and Workers of the Species of Vespa of the World}

1 Posterior ocelli about as close to compound eye as to each other (Fig. 2c) binghami du Buysson

Posterior ocelli closer to each other than to compound eye (Fig. 2d) 
2 Apical margin of clypeus with medial tooth between laterally produced margins (Fig. 2e) ............................... analis Fabricius

Apical margin of clypeus without medial tooth between laterally produced margins (Fig. $2 \mathrm{f})$......

3 Gena more than $1.7 \times$ medial width of compound eye in side view (Fig. 3a), interocellar distance much less than the distance from ocelli to the vertex..... .....4

- Gena less than $1.7 \times$ medial width of compound eye (Fig. 3b), interocellar distance as long or almost as long as the distance from ocelli to the vertex ....

4 Metasomal terga 3-6 black, or at most with narrow apical band on tergum 3 (Fig. 3c) ............................. soror du Buysson

- Metasomal segments 3-6 with orange apical band, tergum 6 mostly orange (as in Fig. 3d) mandarinia Smith

5 Punctures on sides of tergum 2 large, separated by less than one puncture width (Fig. 4a); pronotal carina barely interrupted by pronotal fovea (Fig. 4c)

...6 6

- Punctures on sides of metasomal tergum 2 small, separated by more than one puncture diameter (Fig. 4b); pronotal carina widely interrupted by pronotal fovea (Fig. 4d)...

6 Clypeus mostly black (Fig. 4e); metasomal terga mostly black or at most with yellow apical band on tergum 1 (Fig. 5a) fervida Smith

- Clypeus yellow (Fig. 4f); metasomal terga 1-5 often with apical yellow band (Fig. 5b) luctuosa de Saussure

7 Pretegular carina complete (Fig. 7a); clypeus medially with coarse or large punctures, separated by one puncture diameter or less (Fig. 6c) .

- Pretegular carina incomplete (Fig. 7b); middle of clypeus with small punctures separated more than one puncture diameter (Fig. 6d)

...16

8 Apical margin of clypeus produced into two acute triangular projections (Fig. 8a) ..................................................................9

- Apical margin of clypeus produced into two broadly rounded projections (Fig. 8b)..

...11

9 Vertex black (Fig. 8c) and first three metasomal terga primarily orange-yellow (Fig. 7a) ....................philippinensis de Saussure

- Vertex orange (Fig. 8d) or light brown, if black then orangeyellow coloration restricted to first two metasomal terga (Fig. 7c), or first three metasomal terga black, with at most narrow distal orange-yellow band (Fig. 7d)

10 Metasomal terga never entirely black; metasomal tergum 2 with broad orange-yellow band extending one-third or more across tergum (Fig. 7c and d)...

ducalis Smith

- Metasomal terga all black (Fig. 7f), or metasomal tergum 2 orange-yellow (Fig. 7e), or tergum 2 black with narrow, distal orange-yellow band that extends much less than one-third of tergal width (Fig. 7b)............................tropica (Linnaeus)

11 Metasomal terga 2, 5 and 6 reddish brown or dark brown; tergum 3 and usually tergum 4 mostly yellow, with basal reddish brown band extending medially and two small, lateral reddish brown spots (Fig. 9a and b). orientalis Linnaeus

- Metasomal terga coloration not as above ..............................12

12 Metasomal segment $10.5 \times$ as long as or longer than width in dorsal view (Fig. 9c) .

...13

- Metasomal segment 1 less than $0.5 \times$ as long as wide in dorsal view (Fig. 9d). .14

13 Scutellar punctures small and separated two puncture diameters (Fig. 10a); metasomal tergum 2 mostly black (Fig. 10c) mocsaryana du Buysson

Scutellar punctures large and contiguous or separated by 1 puncture diameter or less (Fig. 10b); metasomal tergum 2 usually reddish brown (Fig. 10d), or if metasomal tergum is mostly black then the vertex is also black..... affinis (Linnaeus)

14 Clypeus strongly bulging medially, strongly convex in side view (Fig. 11a) ..............................................fumida van der Vecht Clypeus not bulging medially, flat or gently curved in side view (Fig. 11b). . .15

15 Metasomal segments 3-5 mostly yellow (Fig. 11c); vertex orange-yellow to red .....................................crabro Linnaeus

Metasomal segments 3-5 mostly dark brown or black, some segments with narrow distal yellow band (Fig. 11d); vertex reddish to dark brown .......................................dybowskii André

16 Clypeus (Fig. 12a) and metanotum with dark markings multimaculata Pérez Clypeus and metanotum without black markings (Fig. 12b and d)

17 Clypeus yellow with black markings (Fig. 12a), or if without markings then metanotum entirely yellow (as in Fig. 12c and d) bellicosa de Saussure

- Clypeus yellow to brown, without black markings (Fig. 12b); metanotum coloration variable (Fig. 12d and e) ...................18

18 Scutellum and metanotum entirely or primarily yellow (Fig. 12d) and vertex black .............................bicolor Fabricius Scutellum and metanotum not primarily yellow (Fig. 12e), or if primarily yellow then vertex only partly black......................19

19 Scutal punctures small, separated by 2-3 puncture diameters (Fig. 13a); metasomal terga 2-5 entirely black..... basalis Smith Scutal punctures large, separated by 1 puncture diameter or less (Fig. 13b); metasomal terga 2-5 not entirely black ...............20

20 Clypeal punctation uniformly distributed ........ simillima Smith Clypeal punctation irregularly distributed, with impunctate

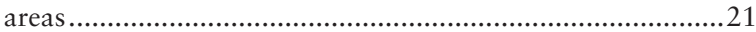

21 Side of prothorax close to spiracle smooth (Fig. 13c); vertex not black; mesothorax and propodeum with same coloration; terga 2 and 5 black or mostly black.............................vivax Smith Side of prothorax close to spiracle rugose (Fig. 13d); without above color combination velutina Lepeletier

\section{World Species of Vespa}

\section{Vespa affinis (Linnaeus)}

Figs. 4d, 9c, 10b, 10d, and 14

Apis affinis Linnaeus, 1764: 417. Holotype female; 'in Calidis regionibus' (UPPSALA).

Vespa affinis Fabricius, 1787: 287. Syntype females; China (COPENHAGEN). Nec Vespa affinis (Linnaeus, 1764).

Vespa unifasciata Olivier, 1792: 677. Type unknown; 'Indes orientales' (repository unknown). Nec Vespa unifasciata Gmelin, 1790.

Vespa alduini Guérin-Méneville, 1831: 264. Holotype male; Indonesia, Maluku, Buru Isl., 'l'île de Bourou, l'une des Moluques' (GENOA).

Vespa bimaculata Guérin-Méneville, 1838: 264. Unnecessary replacement name for Vespa alduini Guérin-Méneville. Nec Vespa bimaculata Geoffroy, 1785, and Vespa bimaculata Olivier, 1792.

Vespa nigripennis de Saussure, 1854: 156. Type unknown; Philippines (repository unknown). Nec Vespa nigripennis Degeer, 1773.

Vespa cincta var. picea du Buysson, 1905 (1904): 537, Lectotype female (designated by van der Vecht, 1959: 214); Papua New Guinea, 'Tupuseleia or Kapakapa' (van der Vecht does not indicate which locality) (GENOVA). 


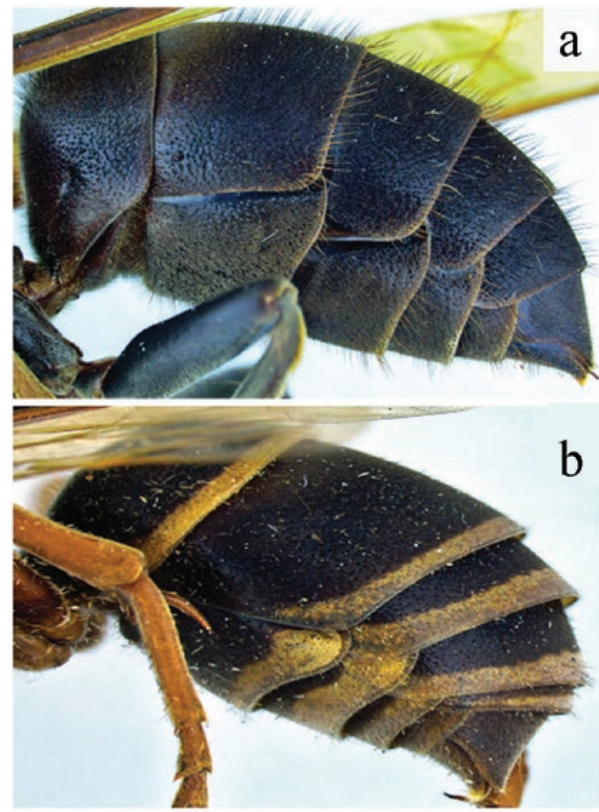

5.
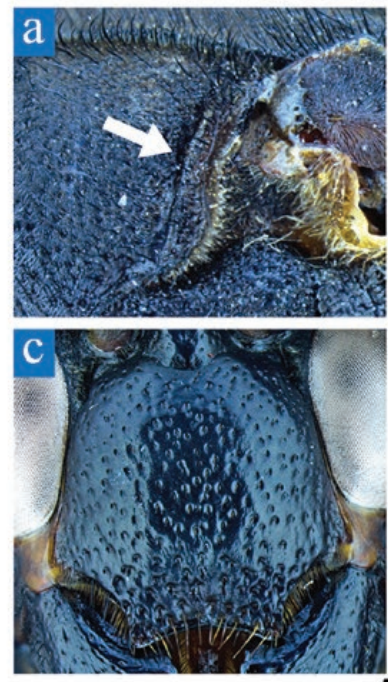

b
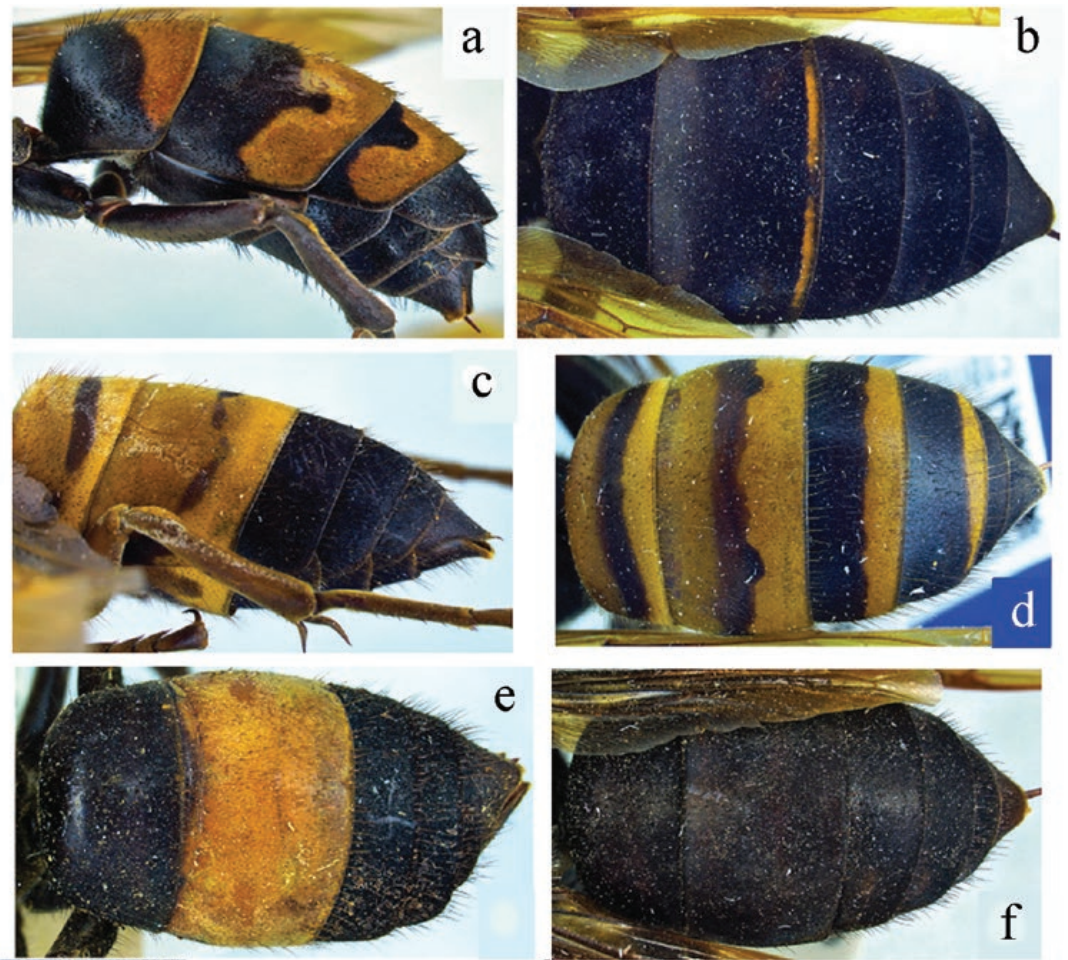

6.
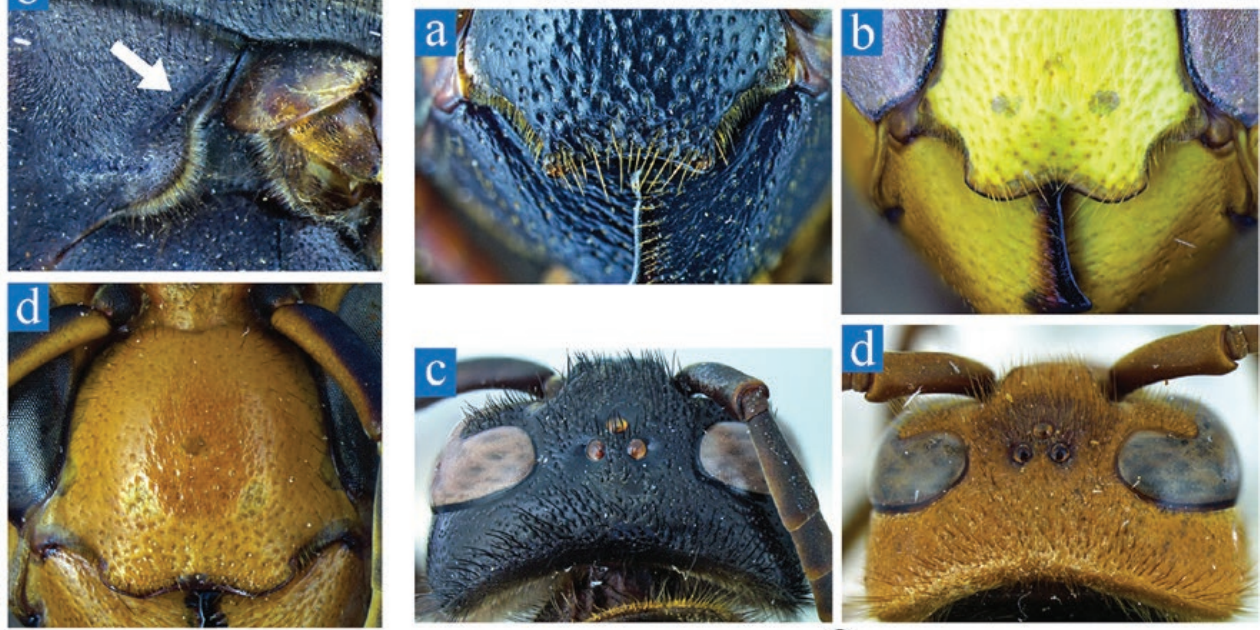

7.
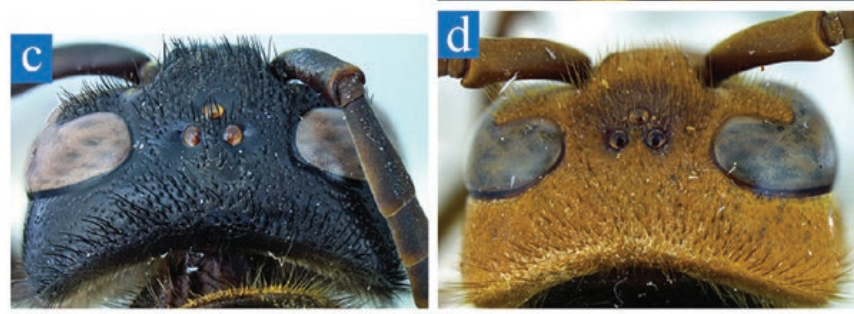

8.

Figs. 5-8. Vespa morphology. Fig. 5. Lateral view of metasomal tergum 2. Fig. 6. (a, b) Lateral view of pretegular pronotal carina. (c, d) Front view of clypeus. Fig. 7. $(a, b)$ Front view of clypeus. (c, d). Dorsal view of vertex. Fig. 8. (a, c) Lateral view of metasoma. (b, d, e) Dorsal view of metasoma. Fig. 5a, V. fervida. Fig. 5b, V. Iuctuosa. Figs. 6a, 6c, 7a, 8a, 8c, V. philippinensis. Figs. 6b, 6d, V. velutina. Figs. 7b, 7e, 7f V. tropica. Figs. 7c, 7d, 8d, V. ducalis. Fig. 8b, V. bicolor.

Vespa indosinensis Pérez, 1910: 8. Lectotype female (designated by van der Vecht, 1957: 28); Vietnam: Annam (PARIS). Given as V. affinis indosinensis by van der Vecht, 1957.

Vespa formosana Sonan, 1927: 125. Lectotype female (designated by Kojima et al. 2011: 45); Taiwan: Taihoku (TAICHUNG).

Vespa affinis var. continentalis Bequaert, 1936: 350. Holotype female; India: Mangalore (WASHINGTON).

Vespa affinis var. hainanensis Bequaert, 1936: 349. Holotype female; China: Hainan Is., 2 mi s Nodoa (CAMBRIDGE).

Vespa affinis nigriventris van der Vecht, 1957: 29. Holotype female; Philippines: Palawan, Puerto Princesa (LOGAN).

Vespa affinis rufonigrans van der Vecht, 1957: 29. Holotype female; Indonesia: Sulawesi, Palu 'North West Celebes' (LEIDEN).
Vespa affinis archboldi van der Vecht, 1957: 32. Holotype female; Indonesia: 'Hollandia' (LEIDEN).

Vespa affinis moluccana van der Vecht, 1957: 32. Holotype female; Indonesia: Saparua I. (LEIDEN).

Vespa affinis alticincta van der Vecht, 1957: 33. Holotype female; New Britain (LONDON-NHM).

Distribution. India, Sri Lanka, Bangladesh, China, Hong Kong, Taiwan, Japan (Ryukyu Is.), Myanmar, Thailand, Laos, Vietnam, Malaysia, Singapore, Indonesia, Papua New Guinea, Philippines, and introduced into Australia, New Zealand, United States.

Discussion. This is one of the smaller bodied hornets, and is common in subtropical and tropical Asia. They are generalist scavengers, 


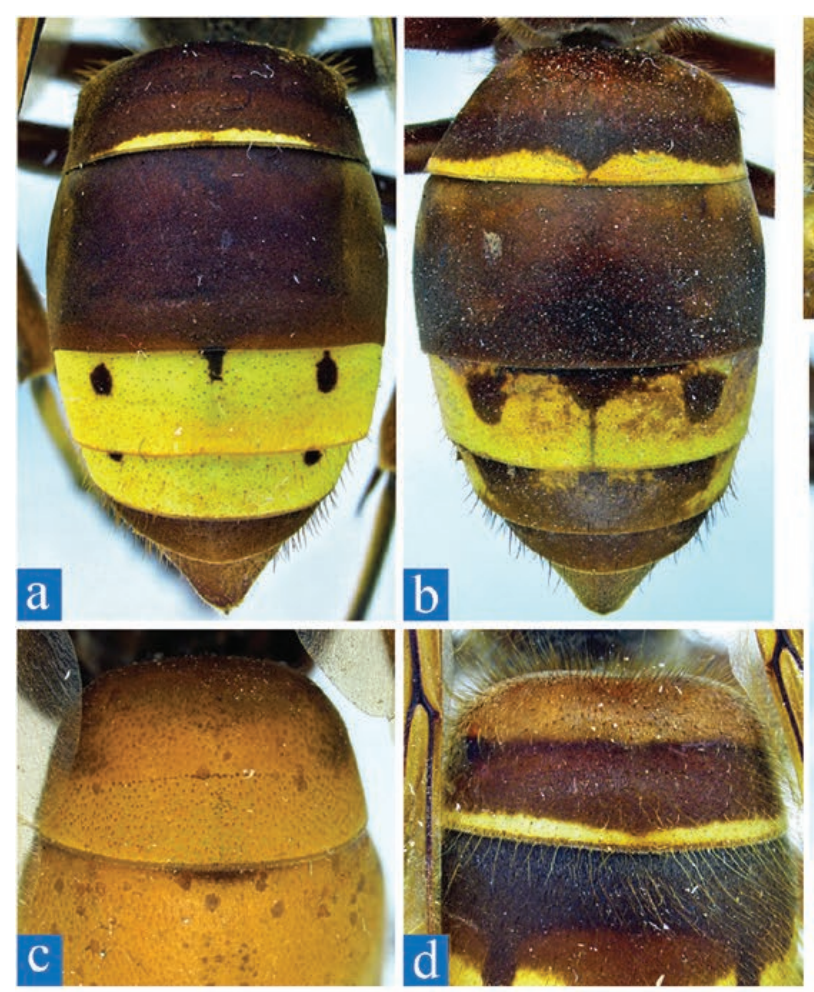

9.
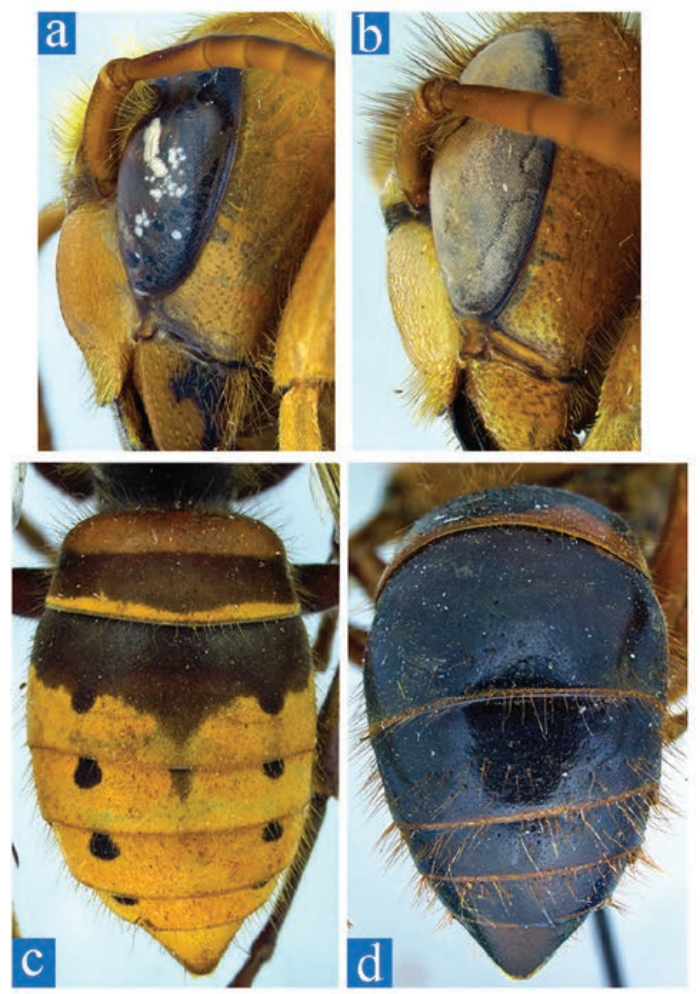

11.
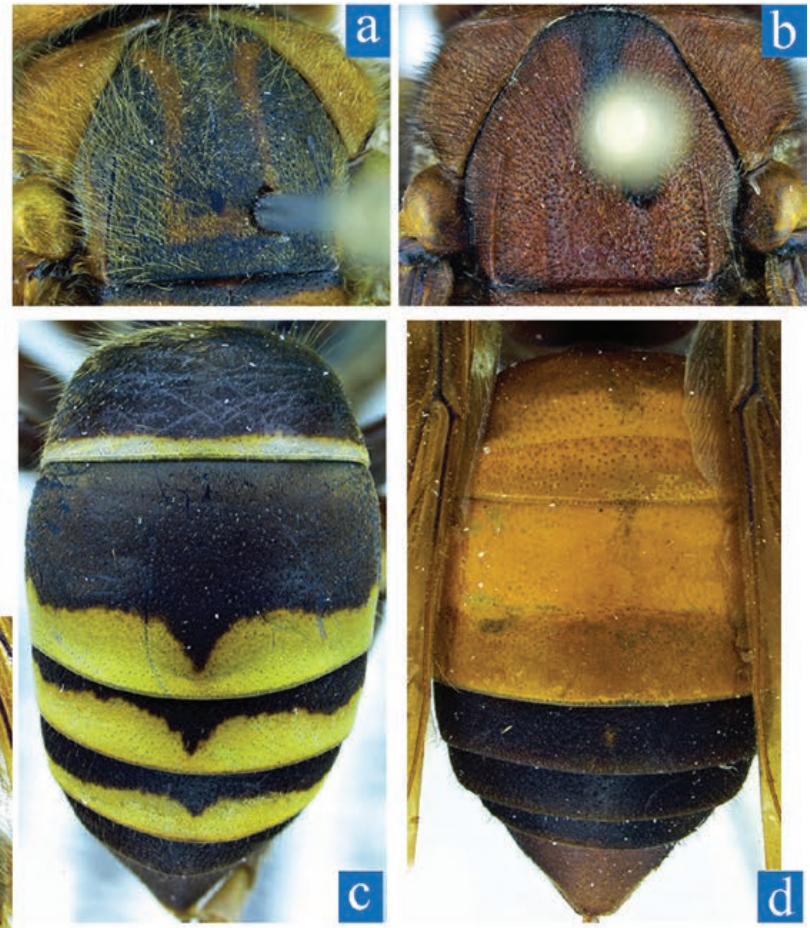

10.
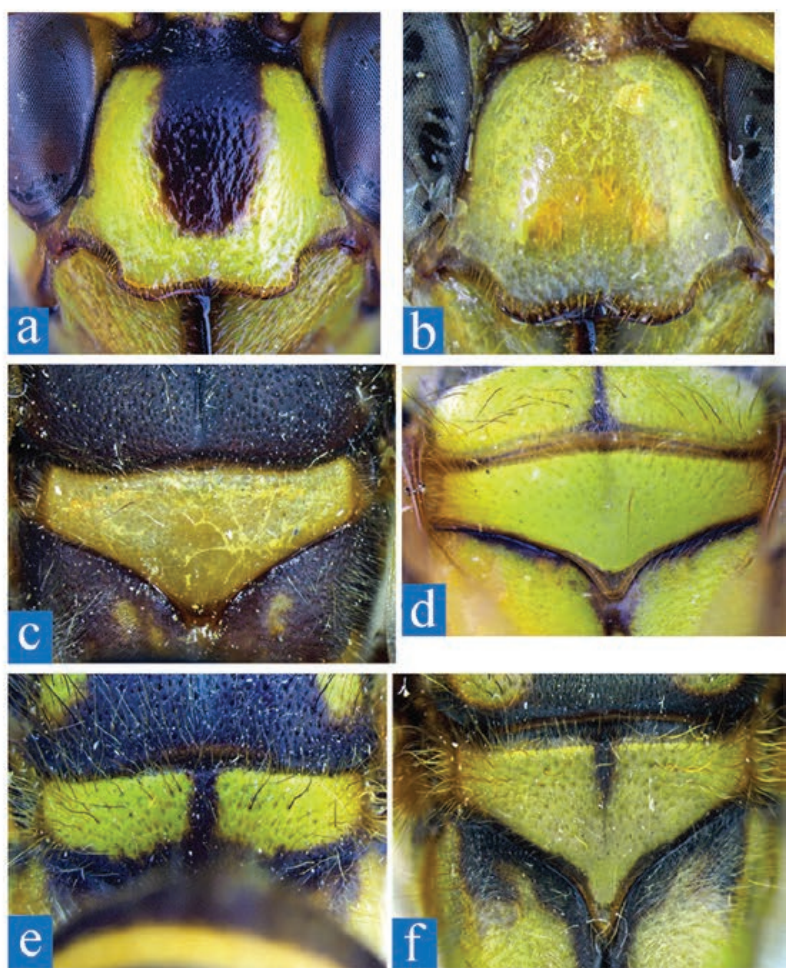

12.

Figs. 9-12. Vespa morphology. (a, b) Dorsal view of metasoma. (c, d) Dorsal view of basal metasomal terga. Fig. 10. (a, b) Dorsal view of scutum. (c, d) Dorsal view of metasoma. Fig. 11. (a, b) Lateral view of head. (c, d) Dorsal view of metasoma. Fig. 12. (a, b) Front view of clypeus. (c-f) Dorsal view of metanotum. Figs. $9 a, 9 b$, V. orientalis. Figs. 9c, 10b, d, V. affinis. Fig. 9d, V. crabro. Figs. 10a, 10c, 11b, 11c, V. mocsaryana. Fig. 11a, V. fumida. Fig. 11d. V. dybowskii. Figs. 12a, 12f, V. multimaculata. Figs. 12b, d, e, V. bicolor. Fig. 12c, V. luctuosa. 


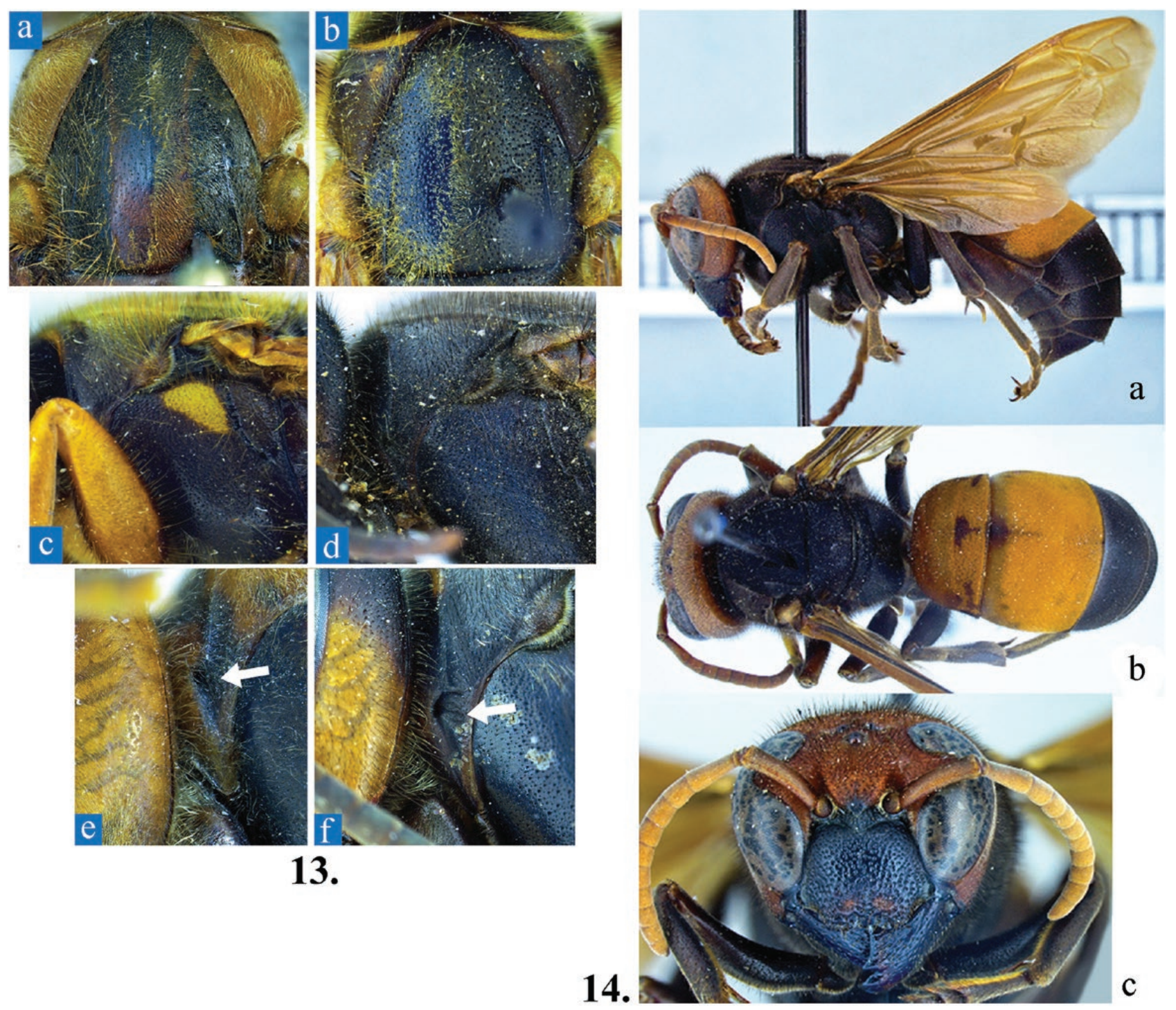

Figs. 13 and 14. Vespa morphology. (a, b) Dorsal view of scutum. (c, d) Lateral view of pronotal lobe. (e, f) Lateral view of anterior pronotal carina. (a) V. basalis. (b) V. ducalis. (c) V. vivax. (d) V. velutina. Fig. 14. V. affinis coloration. (a) Lateral view. (b) Dorsal view. (c) Front view of face.

feeding on nectar, fruit, tree sap, carrion, and insects, including honeybees. Vespa affinis nests are usually built high in trees, but can also be found in shrubs, and on and in buildings (Archer 1997).

\section{Vespa analis Fabricius}

Figs. 2d, 2e, 15, and 16

Vespa analis Fabricius, 1775: 363. Holotype female; 'in Cap. B. S' [in error, probably Java] (LONDON-LS).

Vespa crabro sphinx Christ, 1791: 217. Holotype female (destroyed). Vespa tyrannica Smith, 1857:119. Lectotype female (designated by van der Vecht, 1959: 215); Singapore (LONDON-NHM).

Vespa japonica Smith, 1868: 279. Lectotype male (designated by Kojima, 1997: 20); Japan: 'Hakodadi' (LONDON-NHM). Nec Vespa japonica Radoszkowski, 1857, and Vespa japonica de Saussure, 1858 (=Vespula flaviceps (Smith)).

Vespa parallela André, 1884: lxi. Holotype female; Russia: Vladivostock, 'sur l'Amour' (PARIS).

Vespa insularis Dalla Torre, 1894, Cat. Hym. 9: 147. Replacement name for Vespa japonica Smith.
Vespa tridentata Cameron, 1903: 278. Lectotype female (designated by Kojima, 1997: 20); Japan (LONDON-NHM).

Vespa nigrans du Buysson, 1903: 175. Holotype male; China: 'Yunnam, Tsé-kou' (PARIS).

Vespa parallela var. biroi du Buysson, 1905 (1904): 513. Lectotype female (designated by van der Vecht 1957); Singapore (PARIS).

Vespa analis var. tenebrosa du Buysson, 1905 (1904): 516. Lectotype female (designated by van der Vecht, 1957: 14); Indonesia: Java, Goban (PARIS).

Vespa analis var. (or subsp.) barbouri Bequaert, 1939: 40. Holotype female; India: Sikkim, Teesta Valley (CAMBRIDGE).

Vespa analis var. (or subsp.) kuangsiana Bequaert, 1939: 42. Holotype female; China: 'Kwangsi', (CAMBRIDGE).

Vespa analis eisa Yamane, 1987: 631. Holotype female; Japan: 'Yona, Okinawa-jima' (KAGOSHIMA).

Vespa analis nagatomii Yamane, 1987: 632. Holotype female; Japan: 'Koza-dake, Iriomote-jima' (KAGOSHIMA).

Vespa maguanensis Dong, 2001: 82. Holotype female; China: Yunnan (KUNMING). Synonymized by Carpenter et al. (2011). 


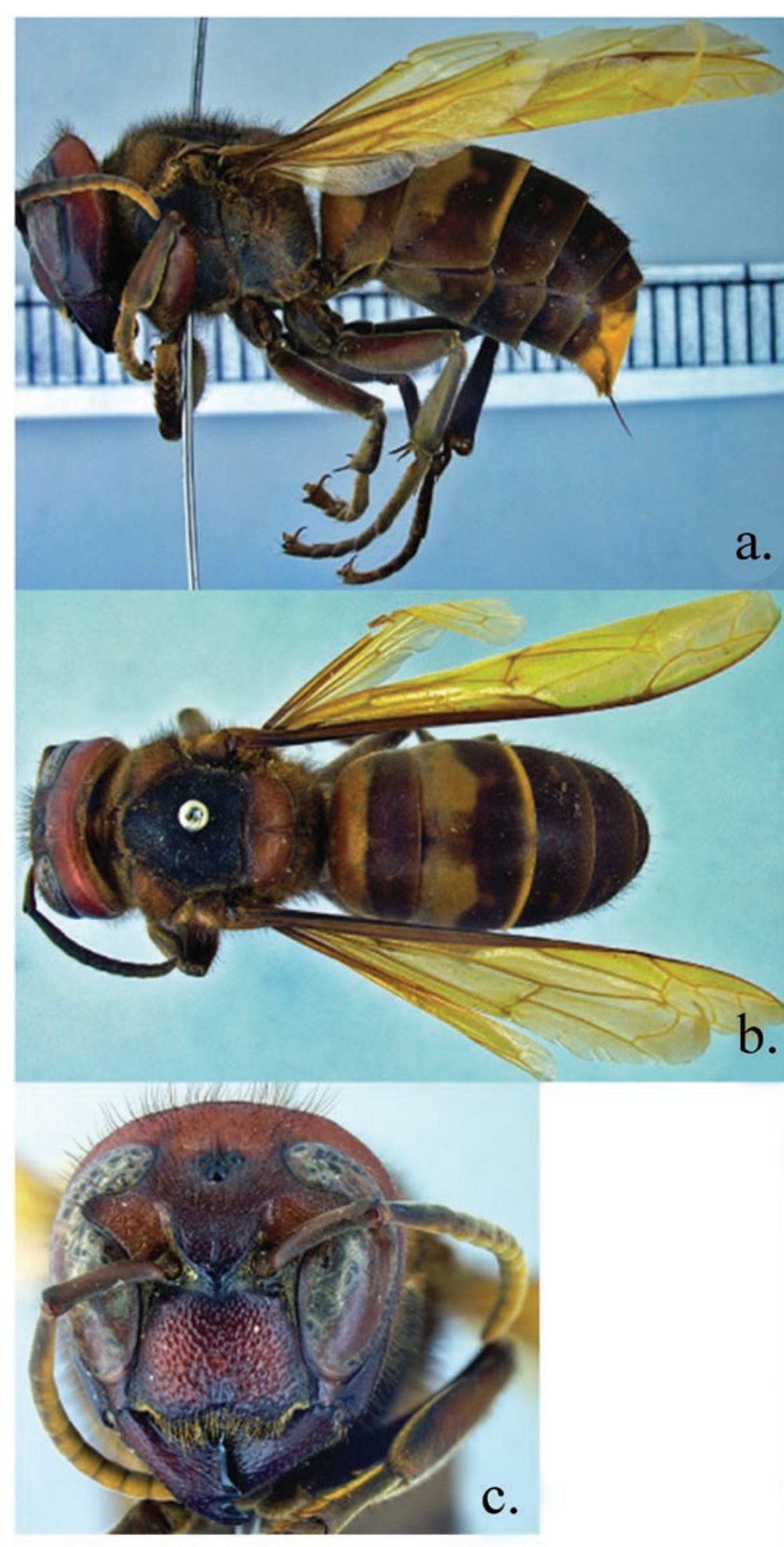

15. Vespa analis "analis" a.

a.
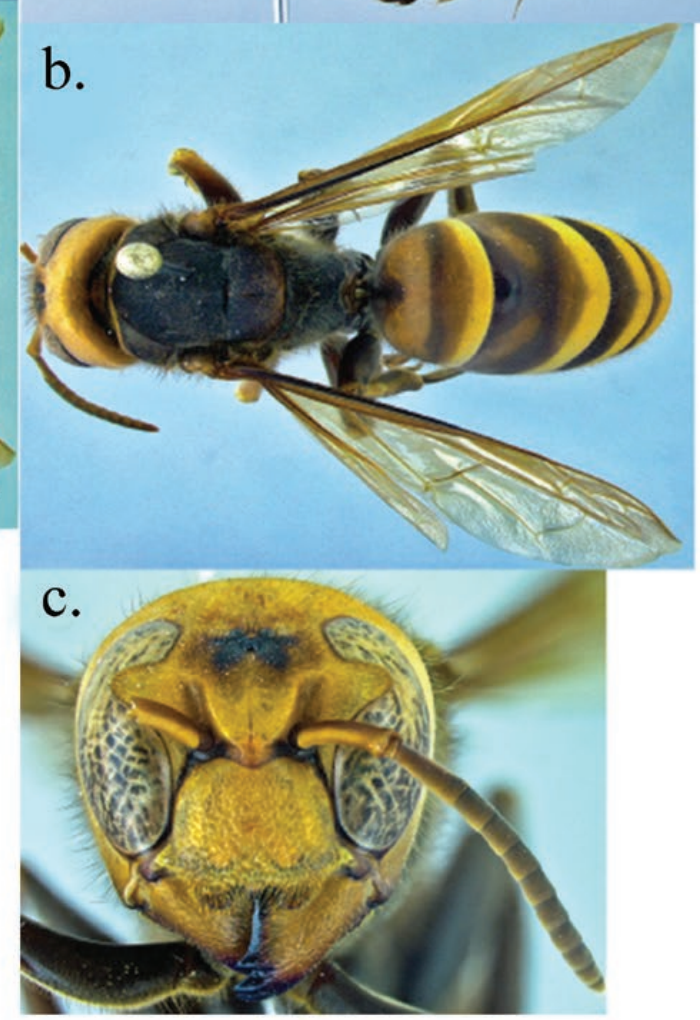

16. Vespa analis "insularis"

Figs. 15 and 16. Vespa analis color forms. (a) Lateral view. (b) Dorsal view. (c) Front view of face.

Vespa hekouensis Dong and Wang, 2003: 407. Holotype female; China, Yunnan, Hekou County (KUNMING). Synonymized by Carpenter et al. (2011).

Distribution. India, Nepal, China, Hong Kong, Taiwan, Korea, Japan, Russia, Myanmar, Thailand, Laos, Vietnam, Malaysia, Malaya, Singapore, Indonesia.

Discussion. This is one of the most widely distributed species of Vespa. Its native range includes tropical Asia and extends north into Japan, Russia, and Korea. Nests are typically built in trees 2-3 m above the ground (Archer 1998b).
Vespa basalis Smith

Figs. $13 \mathrm{a}$ and 17

Vespa basalis Smith, 1852: 46. Holotype female; Nepal: 'Nepaul' (LONDON-NHM).

Vespa obliterata Smith, 1852: 47. Holotype female; 'Northern India' (LONDON-NHM).

Vespa basilis Dover, 1929: 48. Misspelling of Vespa basalis Smith, 1852.

Distribution. Pakistan; India, Nepal, China, Taiwan, Myanmar, Thailand, Laos, Vietnam, Indonesia: Sumatra. 


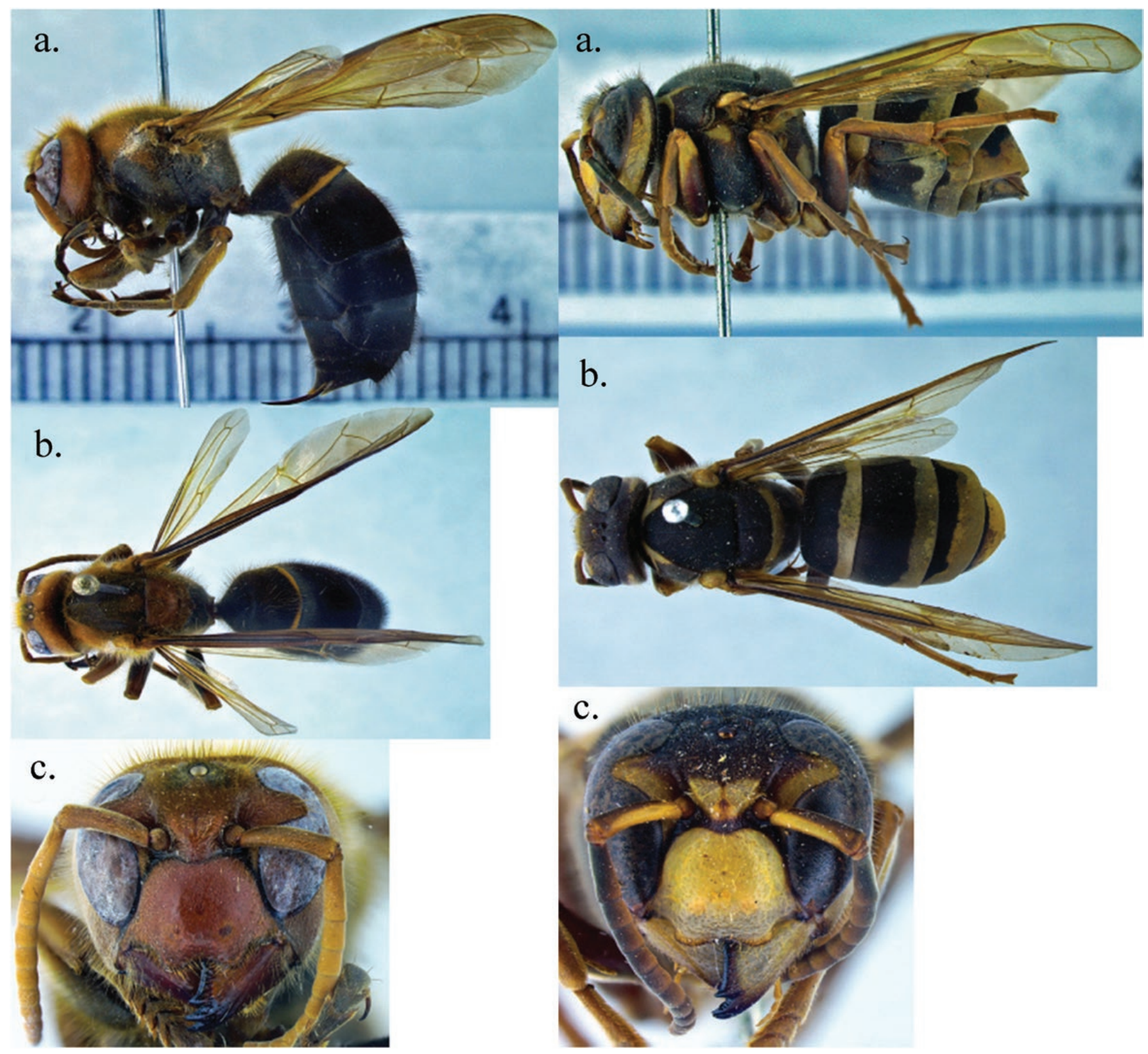

17. Vespa basalis

\section{Vespa bellicosa}

Figs. 17 and 18. Vespa color forms. (a) Lateral view. (b) Dorsal view. (c) Front view of face. Fig. 17. V. basalis. Fig. 18. V. bellicosa.

Discussion. Vespa basalis occurs in forested regions between $500 \mathrm{~m}$ and 1,500 $\mathrm{m}$ (Archer 1999). Nests are built on tree branches, in shrubs, on buildings, in rock crevices, and even in the ground. The mostly dark brown to black metasoma is one of the more distinctive features of $V$. basalis.

\section{Vespa bellicosa de Saussure}

Fig. 18

Vespa bellicosa de Saussure, 1854: 146. Lectotype female (designated by van der Vecht, 1959: 216); 'Java' [error; Sumatra or Borneo] (TURIN).

Distribution. Indonesia: Sumatra, Borneo (Kalimantan, Sarawak). Discussion. This species appears to be associated with lowland tropical forests in Borneo and Sumatra (Archer 1999). It is unlikely that it would become invasive in other regions. It has very distinctive black and yellow banding on the thorax and metasoma. It is not known where $V$. bellicosa build their nests.

\section{Vespa bicolor Fabricius}

Figs. 2f, 8b, 12b, 12d, 12e, 19, and 20

Vespa bicolor Fabricius, 1787: 288. Syntypes, sex not stated; China (COPENHAGEN).

Vespa lutea Coquebert, 1804: 94. Type unknown; 'Massiliae lecta, in navi ex India' (repository unknown).

Vespa auraria var. citriventris du Buysson, 1905 (1904): 552. Lectotype female (designated by van der Vecht, 1959: 218); Sikkim: Padamchen Lingtou, 'Padamtsin à Lingtou' (PARIS).

Distribution. India, Bhutan, Nepal, China, Hong Kong, Myanmar, Thailand, Laos, Cambodia, Vietnam. Introduced into Taiwan. 


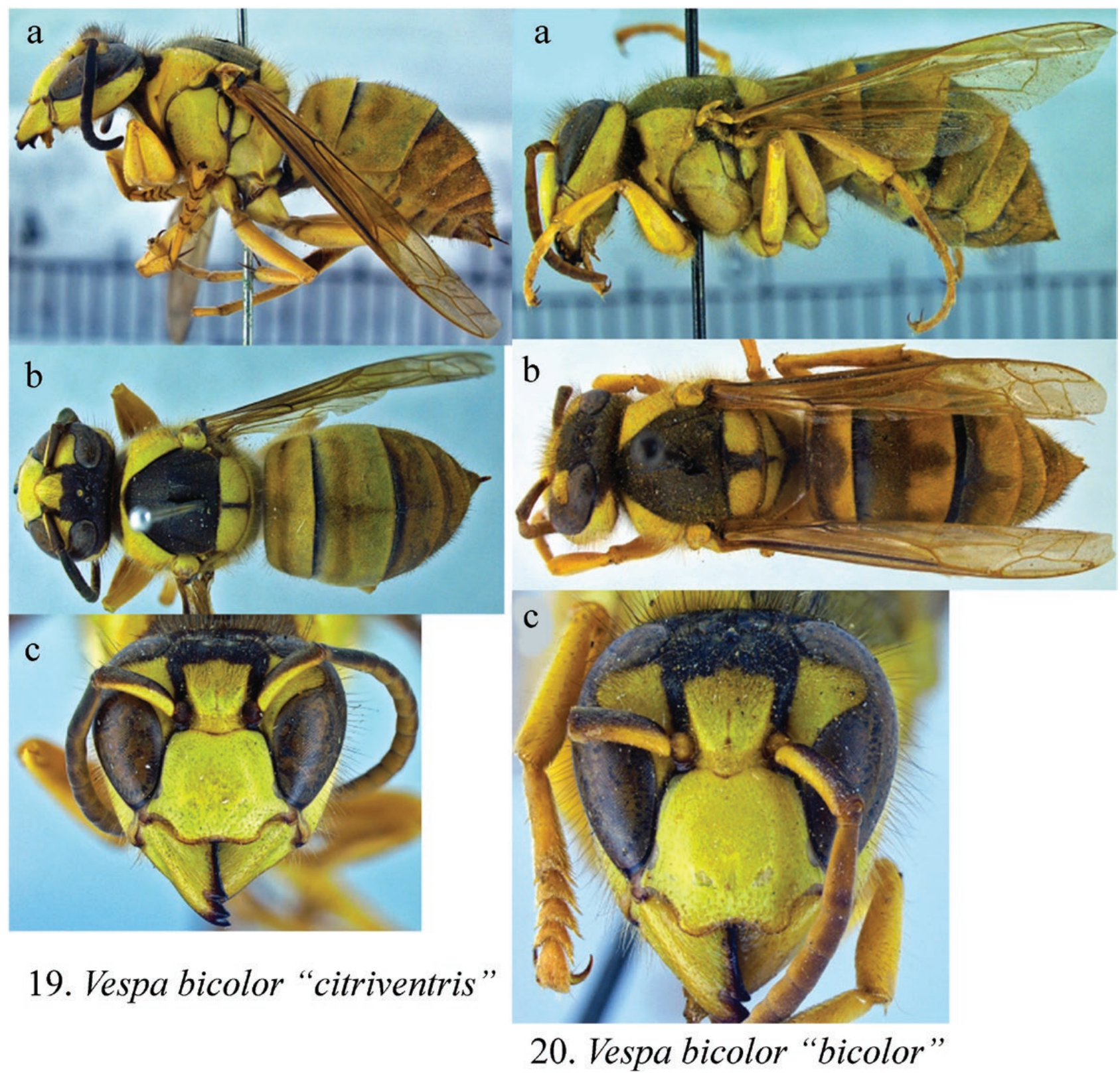

Figs. 19 and 20. Vespa bicolor color forms. (a) Lateral view. (b) Dorsal view. (c) Front view of face.

Discussion. This species of Vespa has a very distinctively marked black and yellow thorax, with a largely yellow metasoma. It preys on honeybees, and one species of Dendrobium orchid exploits this behavior. These orchids produce chemicals that mimic alarm pheromones of Asian (Apis cerana) and European (Apis mellifera) honeybees to attract the hornets to pollinate their flowers (Brodman et al. 2009).

\section{Vespa binghami du Buysson}

Figs. 2c and 21

Vespa binghami du Buysson, 1905 (1904): 523. Lectotype female (designated by van der Vecht, 1959: 210); Myanmar: Toungoo, 'Taungoo Hills' (PARIS).

Vespa suprunenkoi Birula, 1925 (1924): 92. Lectotype female (designated by Kurzenko, 2004: 197); Korea: Sakhalin Is. (ST. PETERSBURG).
Distribution. India, Myanmar, Thailand, Laos, China, Russia, Korea. Discussion. This is a high altitude species, found between $200 \mathrm{~m}$ and 2,000 $\mathrm{m}$ (Archer 1999). Zhang (1989) reported the presence of Vespa binghami in Miocene deposits in Shandong, China, but this identification needs to be verified.

\section{Vespa crabro Linnaeus}

Figs. 1, 2a, 2b, 3b, 3d, 9d, 11b, 11c, and 22-26

Vespa crabro Linnaeus, 1758: 572. Holotype female; 'in Europae' (LONDON-LS).

Vespa vexator Harris, 1776: 128. Holotype female; 'English' (destroyed).

Vespa crabro major Retzius (in Degeer), 1783: 63; Type unknown (repository unknown). 


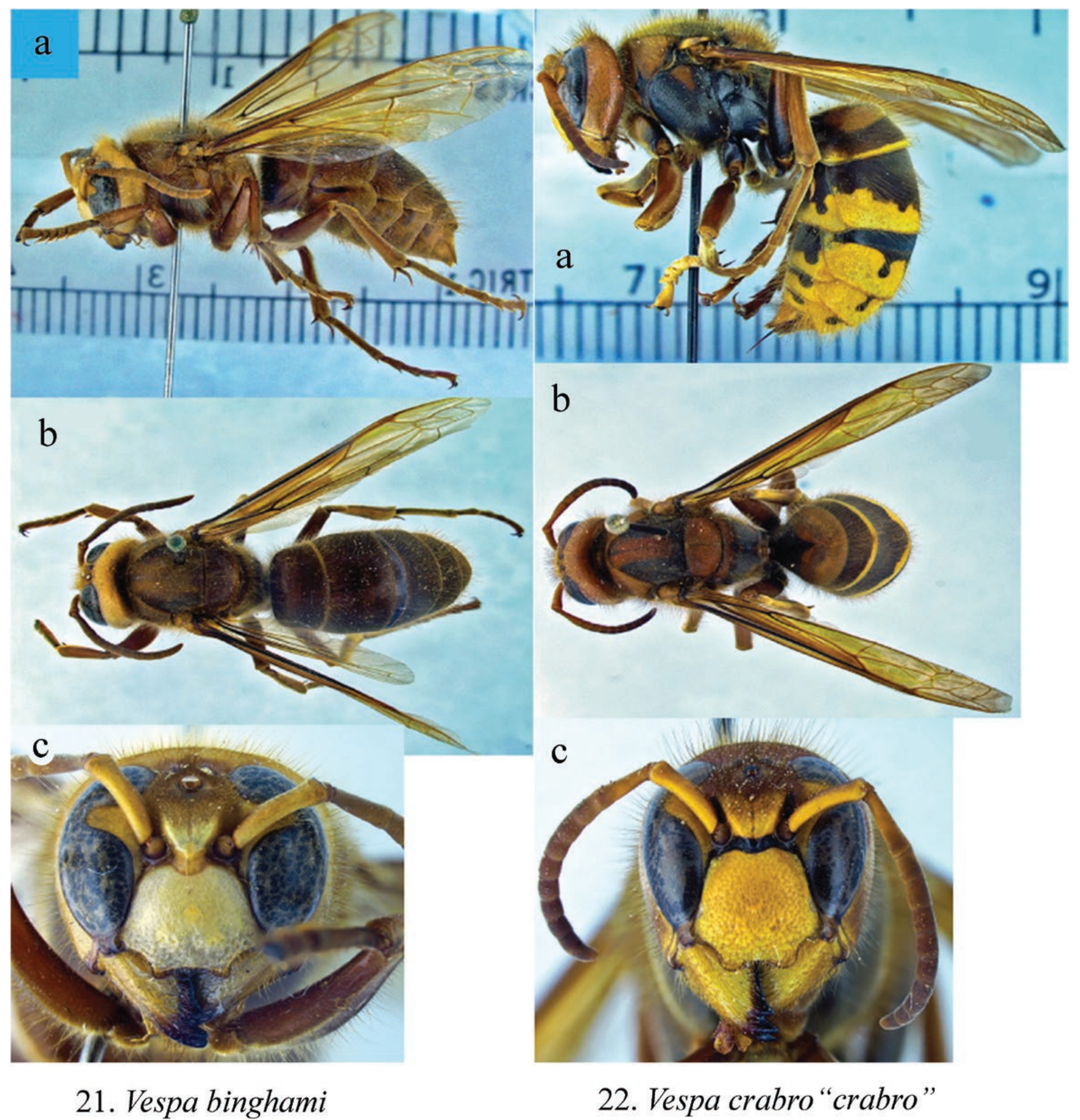

Figs. 21 and 22. Vespa color forms, (a) Lateral view. (b) Dorsal view. (c) Front view of face. Fig. 21. Vespa binghami. Fig. 22. Vespa crabro.

Vespa pratensis Geoffroy (in Fourcroy), 1785: 437; Type unknown; France: 'in Agro Parisiensi' (repository unknown).

Vespa crabro germana Christ, 1791:215. Type unknown (destroyed). Vespa crabroniformis Smith, 1852: 40. Syntype female, male; 'North China' (LONDON-NHM).

Vespa crabro var. borealis Radoszkowski, 1863: 128. Syntype female, male; Russia: 'Pargolova i Osinovoa Roshchi' (repository unknown). Nec Vespa borealis Kirby, 1837, Vespa borealis Zetterstedt, 1840, and Vespa borealis Smith, 1843.

Vespa crabro var. anglica Gribodo, 1892 (1891): 242. Syntype females; England: 'Inghilterra' (GENOA). Nec Vespa anglica Smith, 1843.
Vespa oberthuri du Buysson, 1902: 140; Syntype females; China: 'Chine: Se-Tchouen, Sio-Lou' (PARIS).

Vespa flavofasciata Cameron, 1903: 280. Lectotype female (designated by Kojima, 1997: 21); Japan: 'Nügata (Shinanogawa)' (LONDON-NHM).

Vespa crabro var. tartarea du Buysson, 1905 (1904): 506. Syntype females; Japan: 'Yokohama ... Columbia' (BUDAPEST).

Vespa crabro var. altaica Pérez, 1910: 5. Holotype Female; Russia: Altaï (PARIS).

Vespa crabro var. caspica Pérez, 1910: 6. Holotype Female; Azerbaijan: 'Talysch et Lenkoran, région Caspienne' (PARIS). 


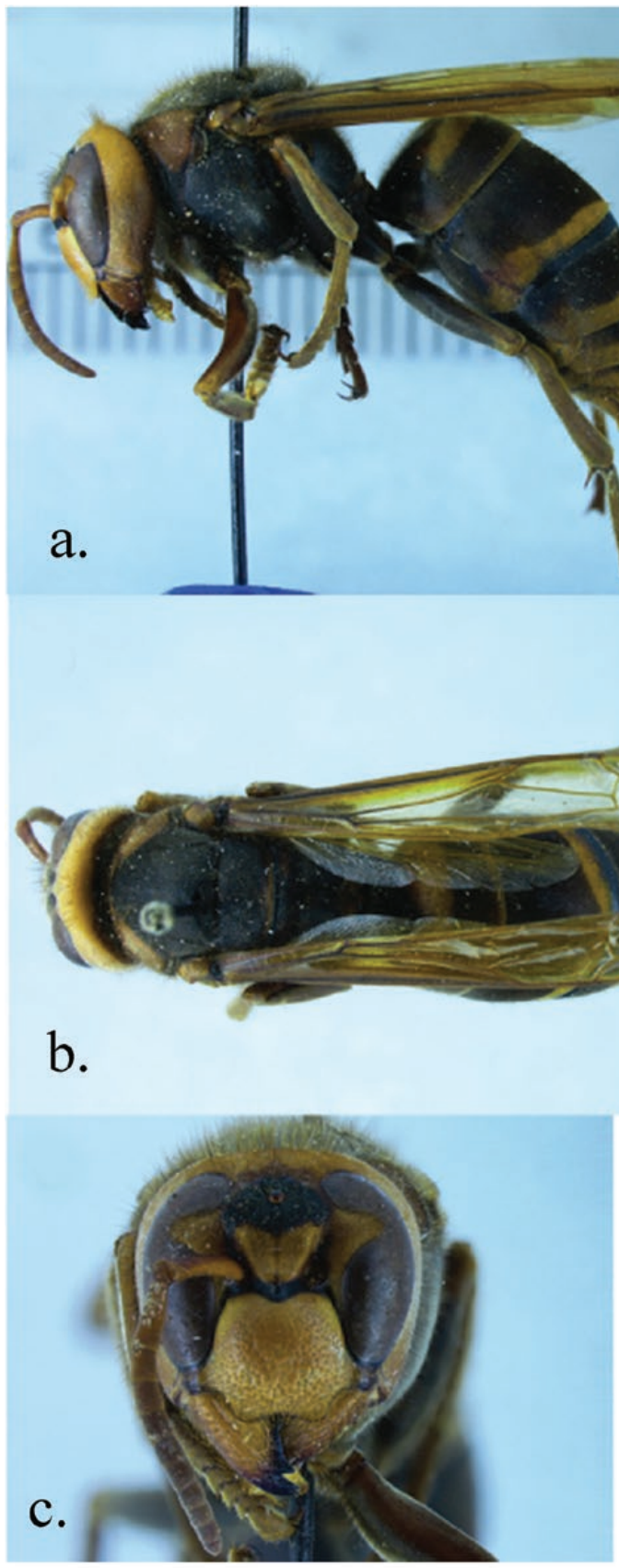

23. Vespa crabro "flavofasciata"
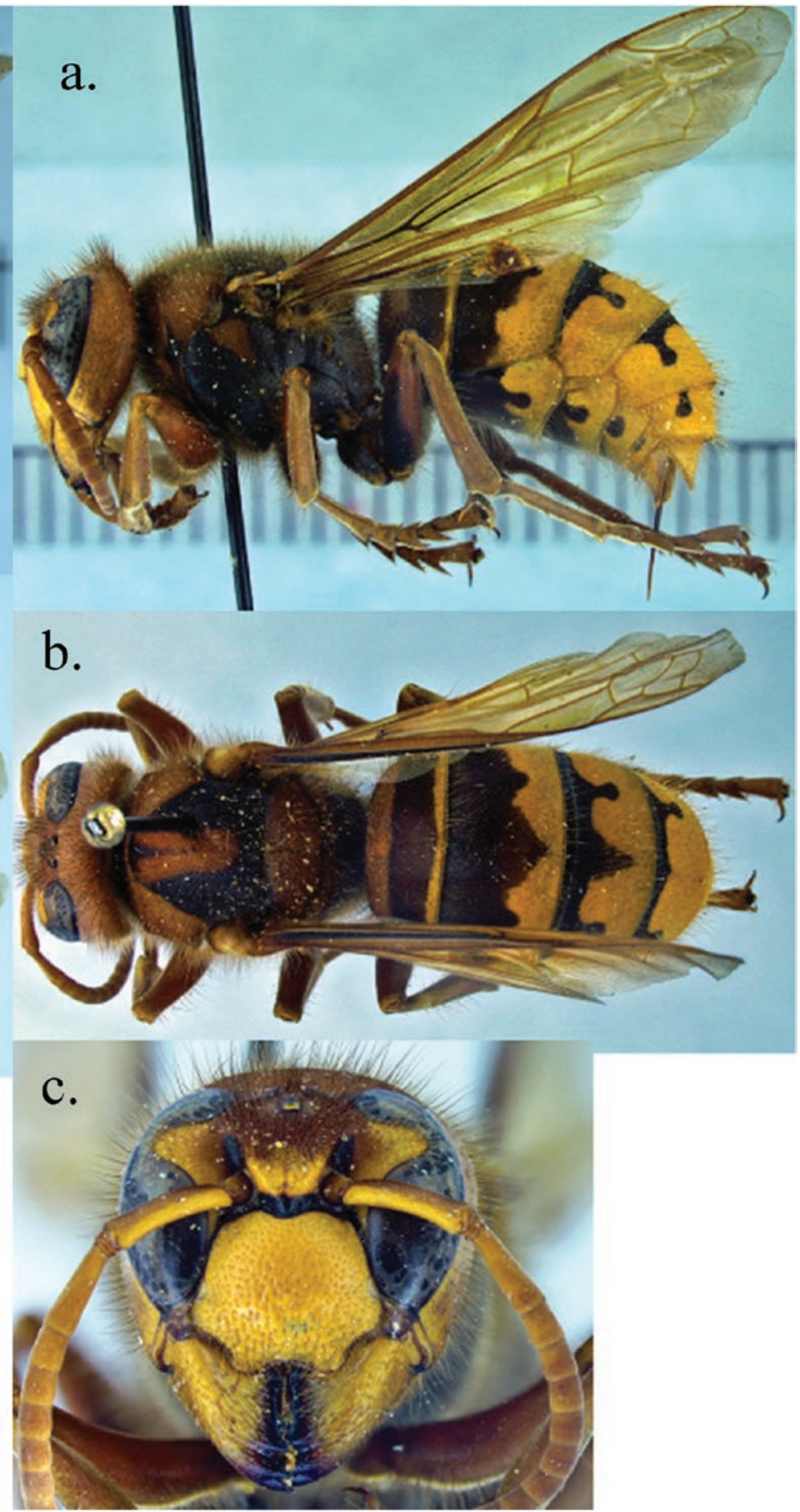

\section{Vespa crabro "germana"}

Figs. 23 and 24. Vespa crabro color forms. (a) Lateral view. (b) Dorsal view. (c) Front view of face.

Vespa crabro nigra Birula, 1925: 55. Syntypes female, male; Russia: West Siberia from Urals to Enisei and slightly after Enisei [in Russian] (ST. PETERSBURG). Nec Vespa nigra Geoffroy, 1785.

Vespa crabro vulgata Birula, 1925: 55. Syntype female, male: west Europe (St. Petersburg).

Vespa crabro meridionalis Birula, 1925: 55. Syntype females: Transcaucasia, north Persia, western part of Transcaspian region (ST. PETERSBURG).

Vespa crabro chinensis Birula, 1925: 55. Syntypes female, male: middle and south China [in Russian] (ST. PETERSBURG). Nec Vespa chinensis Fabricius, 1793.
Vespa crabro var. birulai Bequaert, 1931: 105. Replacement name for Vespa crabro chinensis Birula.

Vespa crabro var. gribodoi Bequaert, 1931: 105. Replacement name for Vespa crabro var. anglica Gribodo.

Distribution. Eurasia, Algeria. Introduced into eastern North America, and Guatemala.

Discussion. Vespa crabro usually nests in sheltered aboveground sites, such as tree hollows, wall voids, beehives, and outhouses (Archer 1993). However, nests have been found in subterranean 


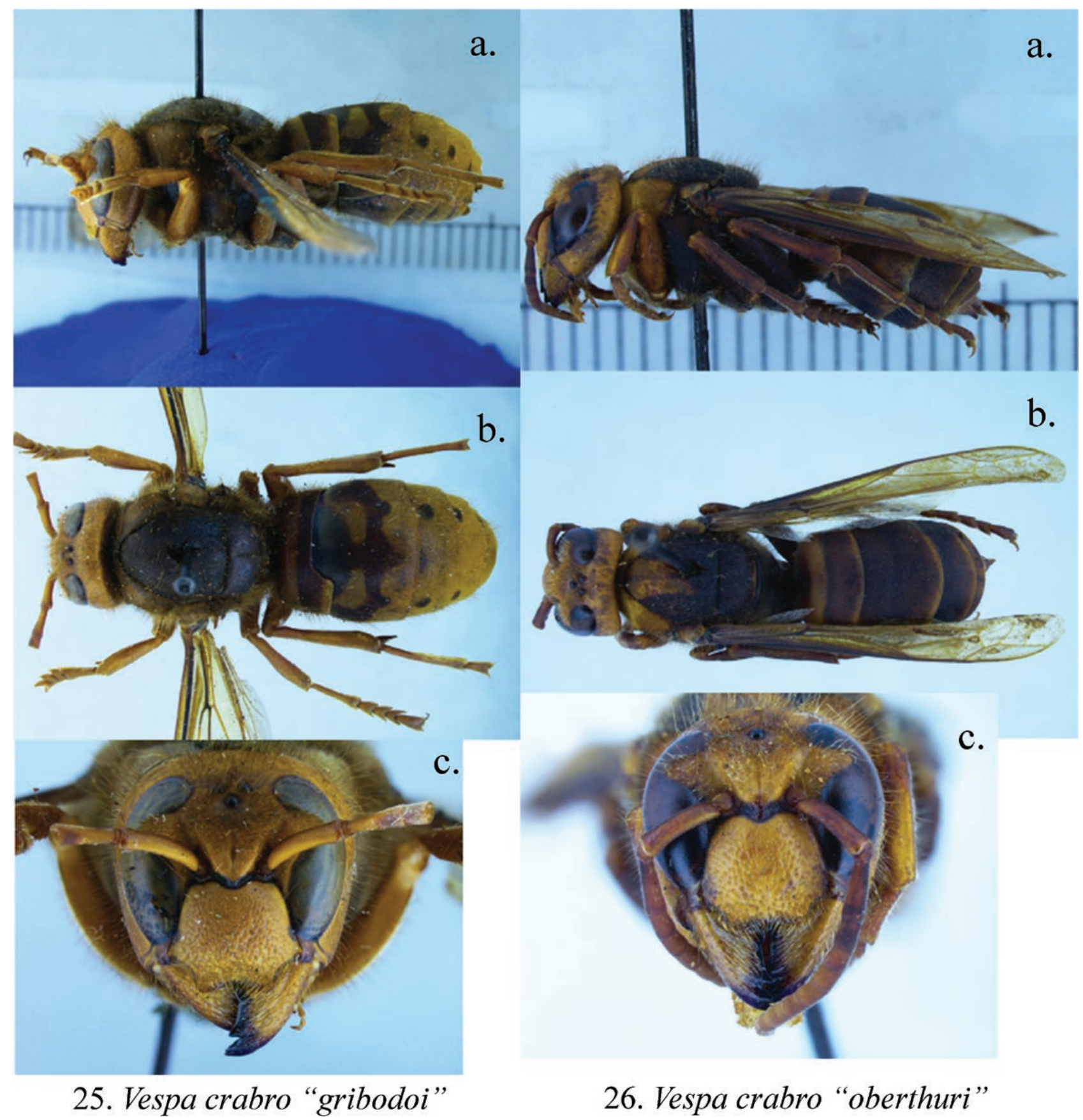

Figs. 25 and 26. Vespa crabro color forms. (a) Lateral view. (b) Dorsal view. (c) Front view of face.

sites as well. This species adapts well to urban and suburban settings. It is widespread in Eurasia, and now eastern North America. The species was first reported in the United States in New York in the 1800s. More recently, the species was recorded from a single worker collected in Guatemala City, Guatemala. It was found in the Entomological Collection of the Universidad del Valle de Guatemala (Landolt et al. 2010). There is no evidence that the species is established in Guatemala. Nests are built in cavities in trees or buildings.

\section{Vespa ducalis Smith}

Figs. $7 \mathrm{c}, 7 \mathrm{~d}, 8 \mathrm{~d}, 13 \mathrm{~b}, 27$, and 28
Vespa ducalis Smith, 1852: 39. Syntypes female, male; China: 'Teintung, near Nigo-po-foo' (LONDON-NHM).

Vespa ducalis var. pulchra du Buysson, 1905 (1904): 519. Lectotype female (designated by van der Vecht, 1959: 224); Japan: Yokohama (PARIS).

Vespa matsumurai Sonan, 1935: 370. Holotype female; Japan: Tokyo (TAICHUNG).

Vespa esakii Sonan, 1935: 371. Holotype female; Japan: Tsushima Is., 'Izuhara' (FUKUOKA).

Vespa tropica loochooensis Bequaert, 1936: 343. Holotype female; Japan: Iriomote Is., Ryukyu Archipelago (CAMBRIDGE). 


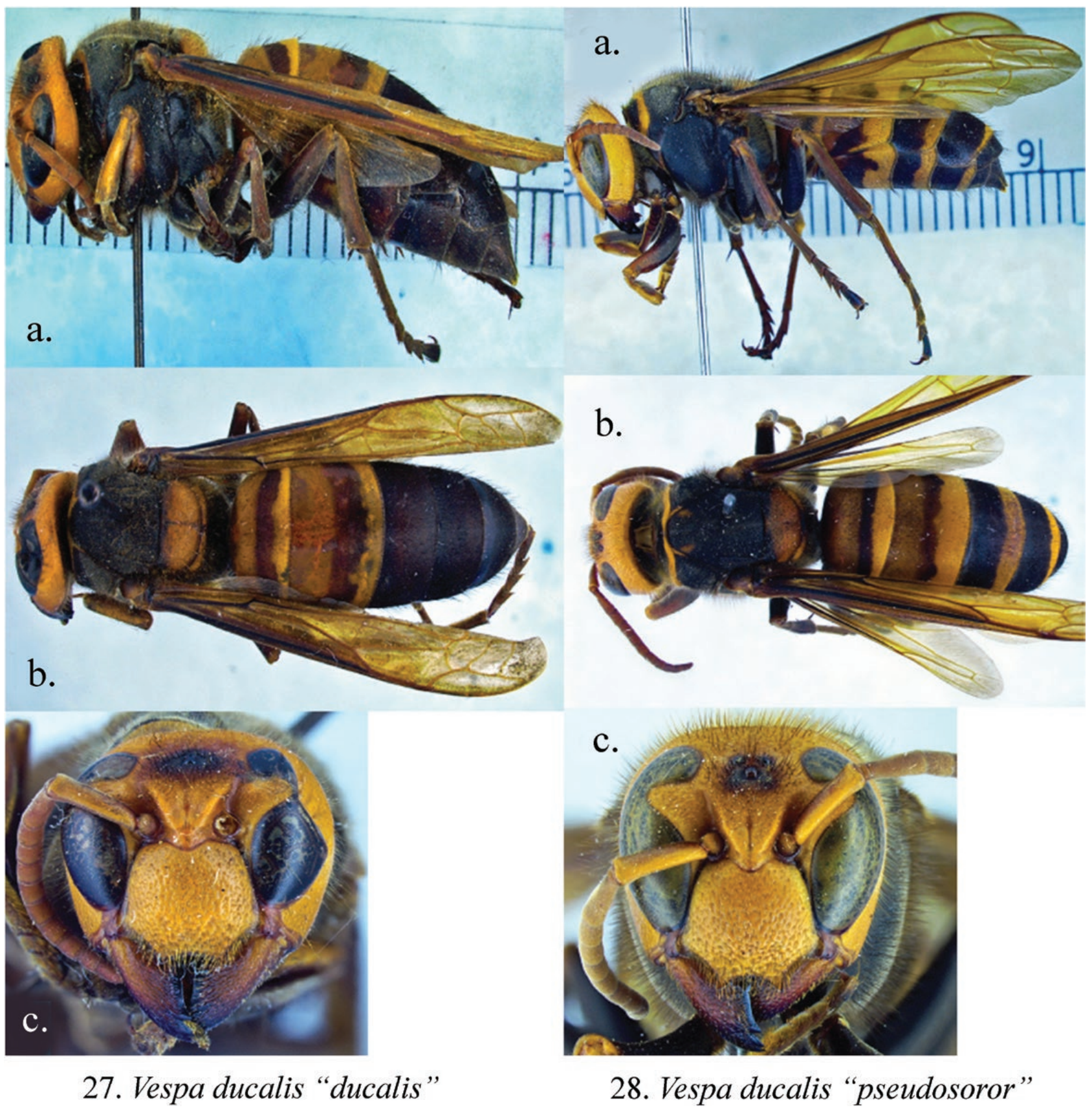

Figs. 27 and 28. Vespa ducalis color forms. (a) Lateral view. (b) Dorsal view. (c) Front view of face.

Vespa tropica pseudosoror van der Vecht, 1959: 224. Holotype female; Vietnam: Annam, Tourane, 1,000 m (PARIS).

Distribution. India, Sikkim, Nepal, Myanmar, Thailand; Laos, Vietnam, China, Hong Kong, Hainan; Taiwan; Russia: Korea; Japan, including Ryukyu Is.

Discussion. This species of Vespa is quite different from the others. It preys on nests of other paper wasps, feeding the paper wasp pupae and larvae to their larvae. Their colonies are small with an average of 50 individuals, and nests are built underground, in tree hollows, and even in attics (Archer 1991).

\section{Vespa dybowskii André}

Figs. 11b, 11d, and 29
Vespa dybowskii 'Rad. in litt'. André, 1884: 582. Holotype female; Russia: 'Sibérie' (PARIS?).

Vespa dubowskii; Dalla Torre, 1904: 65. Misspelling of Vespa dybowskii André.

Vespa walkeri du Buysson, 1905 (1904): 539. Syntype female, male; China: 'Haï-ning' (LONDON-NHM).

Vespa dybowskii mutata Ma, 1937: 30. Holotype male; China: 'Hangchow' (HANGZHOU, destroyed?).

Distribution. Myanmar, China (Tibet, Zhejiang), Russia (Siberia, Primorsky), Korea, Japan.

Discussion. This species is relatively rare in collections. It is a nest parasite of Vespa crabro and V. simillima. Vespa dybowskii queens take over the nests of these species and use the host workers to raise their own brood (Martin et al. 2008) 


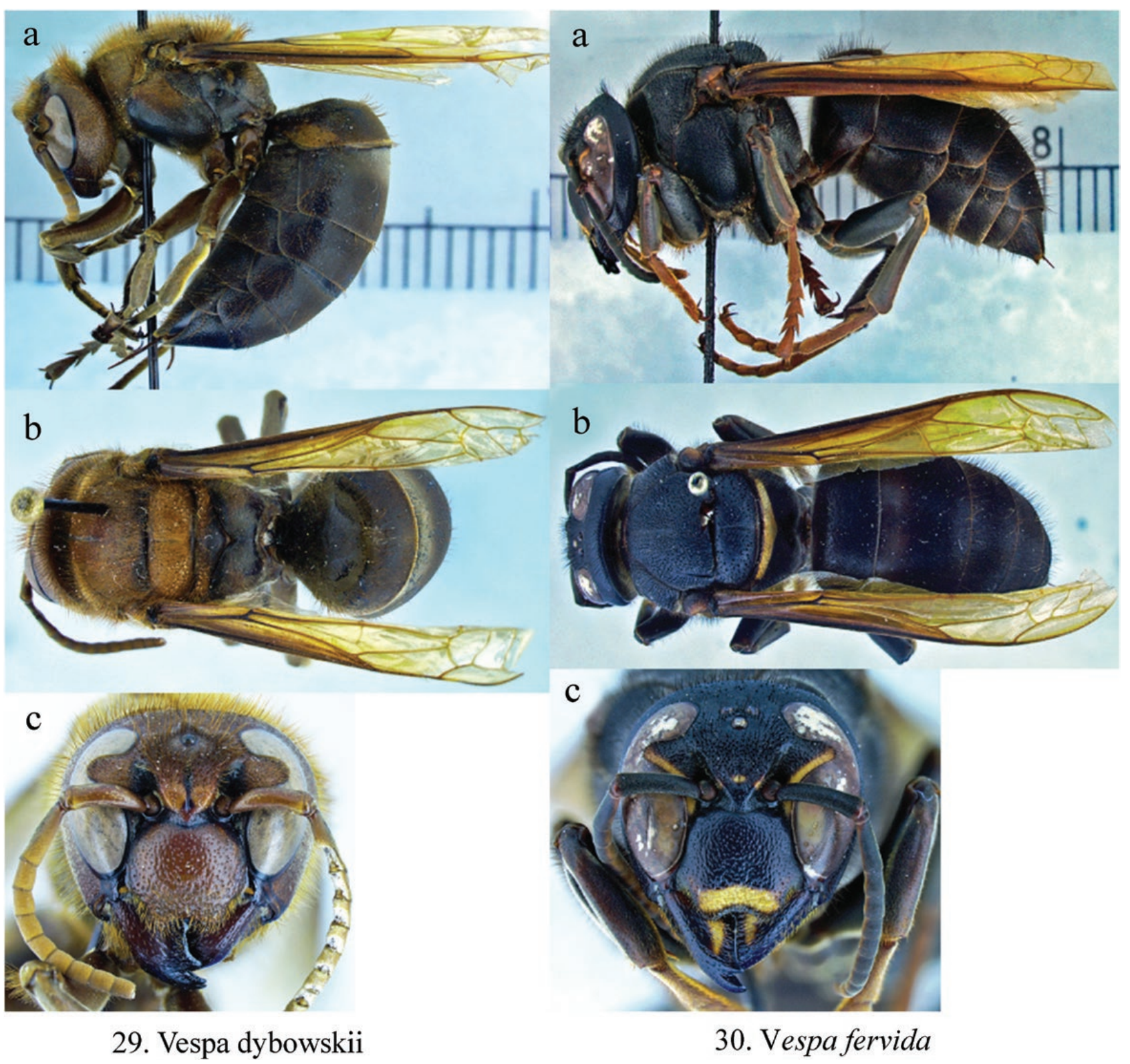

Figs. 29 and 30. Vespa color forms, (a) Lateral view. (b) Dorsal view. (c) Front view of face. Fig. 29. V. dybowskii. Fig. 30. V. fervida.

\section{Vespa fervida Smith}

Figs. 4a, 4e, 5a, and 30

Vespa fervida Smith, 1858: 23. Holotype female; Indonesia: Sulawesi, 'Celebes' (LONDON-NHM).

Distribution. Indonesia (Sulawesi, Salayar, Buton).

Discussion. This species has a very limited distribution on Sulawesi and nearby islands in Indonesia. They build nests in forested areas, and around human habitations, nests have been found under leaves and on fences. It is a small-bodied, dark-colored Vespa. Overall, its biology is poorly known (Matsuura and Yamane 1984, Archer 1999)

\section{Vespa fumida van der Vecht}

Fig. 31

Vespa variabilis du Buysson, 1905 (1904): 522. Lectotype female (designated by van der Vecht, 1959: 228); China: Mou-pin (PARIS). Nec Vespa variabilis Fabricius, 1781.
Vespa variabilis fumida van der Vecht, 1959: 228. Holotype female; India: 'British Bootan', Padong' (PARIS).

Distribution. India, Bhutan, Nepal, Myanmar, China (Sichuan, Yunnan, Hubei, Fujian).

Discussion. Vespa fumida is found at high altitudes between $600 \mathrm{~m}$ and 3,000 m across northeastern India into China (Archer 1999). Otherwise, little is known about its biology.

\section{Vespa luctuosa de Saussure}

Figs. 4c, 4f, 5b, 12c, and 32

Vespa luctuosa de Saussure, 1854: 143. Holotype male; Philippines (LONDON-NHM).

Vespa bellicosa var. semperi du Buysson, 1905 (1904): 542. Lectotype female (designated by van der Vecht, 1957: 48). Philippines: Camiguin Is., 'Comigu' (VIENNA).

Vespa luctuosa var. luzonensis Bequaert, 1934: 5. Holotype female; Philippines: Luzon (BERLIN). 


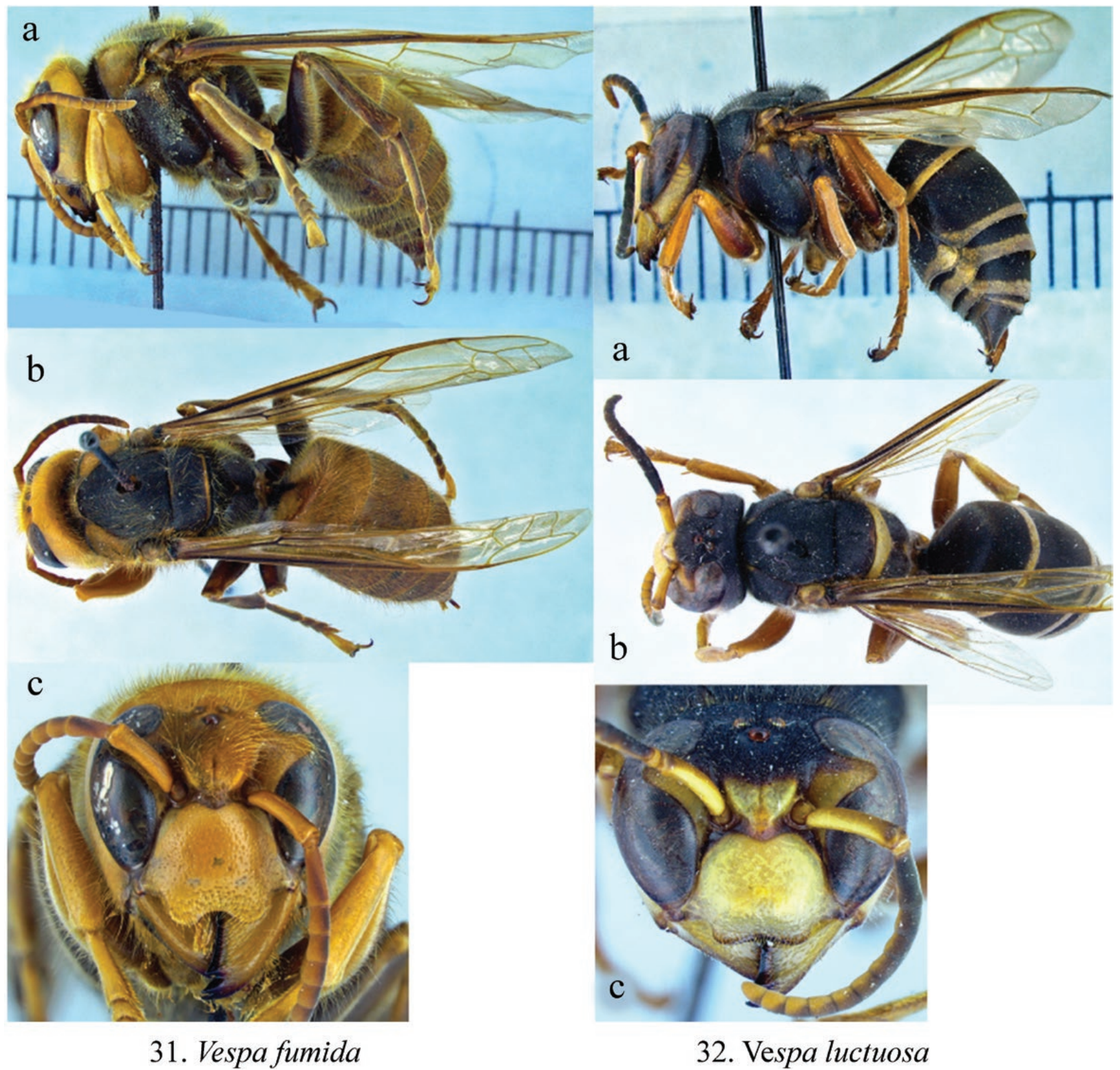

Figs. 31 and 32. Vespa color forms, (a) Lateral view. (b) Dorsal view. (c) Front view of face. Fig. 31. Vespa fumida. Fig. 32. V. luctuosa.

Vespa luctuosa negrosensis Kojima and Tano, 1985: 436. Holotype female; Philippines: 'Mambucal, Negros Is'. (TOKYO). Synonymized by Archer (1999).

Distribution. Philippines.

Discussion. This species has been found nesting in forested regions between 200 and 1,500 m elevation. The nests are built on tree branches, sometimes as high as $20 \mathrm{~m}$ above the ground (Archer 1999).

\section{Vespa mandarinia Smith}

Figs. 33-35

Vespa mandarinia Smith, 1852: 38. Holotype female; China: 'Teintung, near Ning-po-foo' (LONDON-NHM).

Vespa magnifica Smith, 1852: 45. Syntype females; Nepal 'Nepaul' (LONDON-NHM, OXFORD).
Vespa japonica Radoszkowski (in Motschulsky), 1857: 410. Type?; Japan, 'Japon' (ST. PETERSBURG).

Vespa bellona Smith, 1871: 248.Holotype female; China: 'Yunan' (LONDON-NHM).

Vespa magnifica var. latilineata Cameron, 1903: 278. Holotype female; Japan: 'Hitoyoshi' (LONDON-NHM).

Vespa mandarina; Dalla Torre, 1894: 149. Misspelling of Vespa mandarinia Smith.

Vespa magnifica var. nobilis Sonan, 1929: 140. Holotype female; Taiwan: 'Musha' (TAICHUNG).

Vespa magnifica sonani Matsumura, 1930: 1. Lectotype female (designated by Kojima, 1997: 22); Taiwan: 'Sina' (SAPPORO).

Distribution. India, Sri Lanka, Bhutan, Nepal, Myanmar, Thailand, Laos, Vietnam, Malaysia, Malaya, China, Hong Kong, Taiwan, eastern Russia, Korea, Japan (including Ryukyus). 


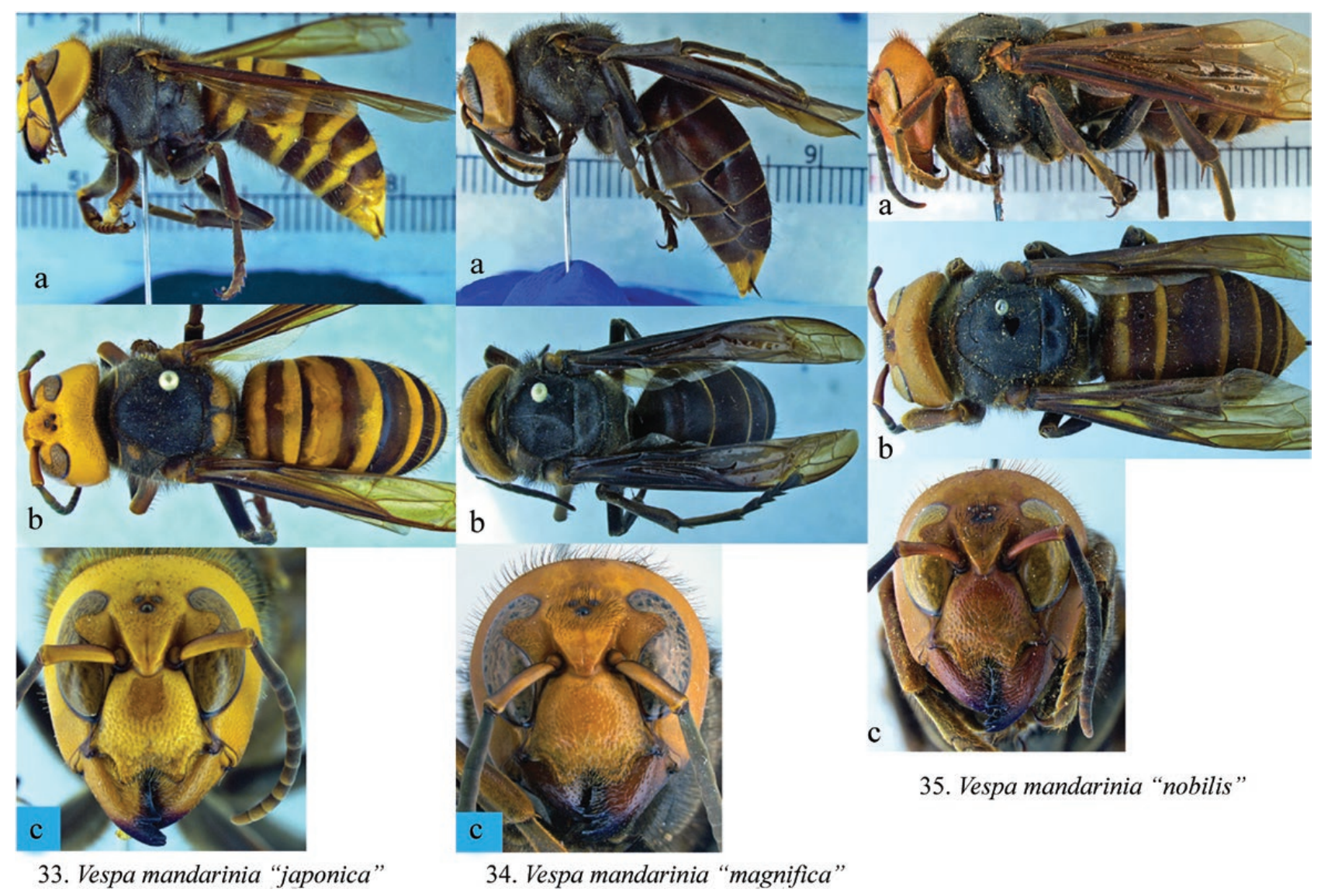

Figs. 33-35. Vespa mandarina color forms. (a) Lateral view. (b) Dorsal view. (c) Front view of face.

Discussion. This species occurs in hilly regions. It nests in the ground, starting with mammal burrows or decayed root cavities, enlarging them as the colony develops (Archer 1995, 2008). This is another species of Vespa that preys on honeybees. Zhang (1989) reported the presence of Vespa magnifica in Miocene deposits in Shandong, China. This identification needs to be verified. This species has also been introduced into the Pacific Northwest, where nests have been found recently (Anonymous 2020).

\section{Vespa mocsaryana du Buysson}

Figs. 10a, 10c, 36, and 37

Vespa mocsaryana du Buysson, 1905 (1904): 537. Syntype females, males; India: Malaysia: 'Presqu'ile de Malacca: Pérak; India: Sikkim; Assam: Khasia Hills; Myanmar: Tenasserim: Thagatâ' (BUDAPEST, VIENNA, LONDON-NHM, GENOVA).

Distribution. India: Meghalaya, Sikkim, Assam; China: Sichuan, Anhui, Fujian, Hong Kong; Myanmar; Thailand; Laos; Vietnam; Malaysia: Peninsular Malaysia; Indonesia: Sumatra.

Discussion. Not much is known about the biology of this species. It occurs in montane forests in southern Asia. Nests have been found in a shrub and on a ceiling (Archer 2008).

\section{Vespa multimaculata Pérez}

Figs. 4b, 12a, 12f, 38, and 39

Vespa annulata Smith, 1858: 116. Lectotype female (designated by Carpenter and Kojima 1997); Malaysia: Borneo: Sarawak (OXFORD). Nec Vespa annulata Rossi, 1790.
Vespa multimaculata Pérez, 1910: 14. Lectotype female (designated by van der Vecht 1957); Brunei (PARIS).

Vespa luctuosa var. malayana Bequaert, 1934: 4. Replacement name for Vespa annulata Smith.

Vespa multimaculata pendleburyi van der Vecht, 1957: 43; Holotype female; Malaysia: 'near Jitra, Kedah', (LEIDEN).

Distribution. Thailand; Laos; Malaysia: Peninsular Malaysia, Sarawak, Sabah; Singapore; Indonesia: Sumatra, Borneo (KALIMANTAN); Brunei.

Discussion. Vespa multimaculata is found in lowland and montane forests in Southeast Asia (Archer 1999, 2011), and is unlikely to disperse to temperate regions. Martin (1995) found nests in the ground beneath the roots of a tree.

\section{Vespa orientalis Linnaeus}

Figs. 9a, 9b, and 40

Vespa orientalis Linnaeus, 1771: 540. Holotype female; 'Oriente' (LONDON-LS).

Vespa turcica Drury, 1773: 74. Type unknown; Turkey: Smyrna (destroyed?).

Vespa quadripunctata Forskål, 1775: 84; Type?; Egypt: Cairo, 'Kahirae' (COPENHAGEN?).

Vespa crabro fusca Christ, 1791: 216. Type unknown; Turkey: 'Smirna' (destroyed).

Vespa aegyptiaca Vallot, 1802: 170; Type unknown; Egypt (repository unknown). 


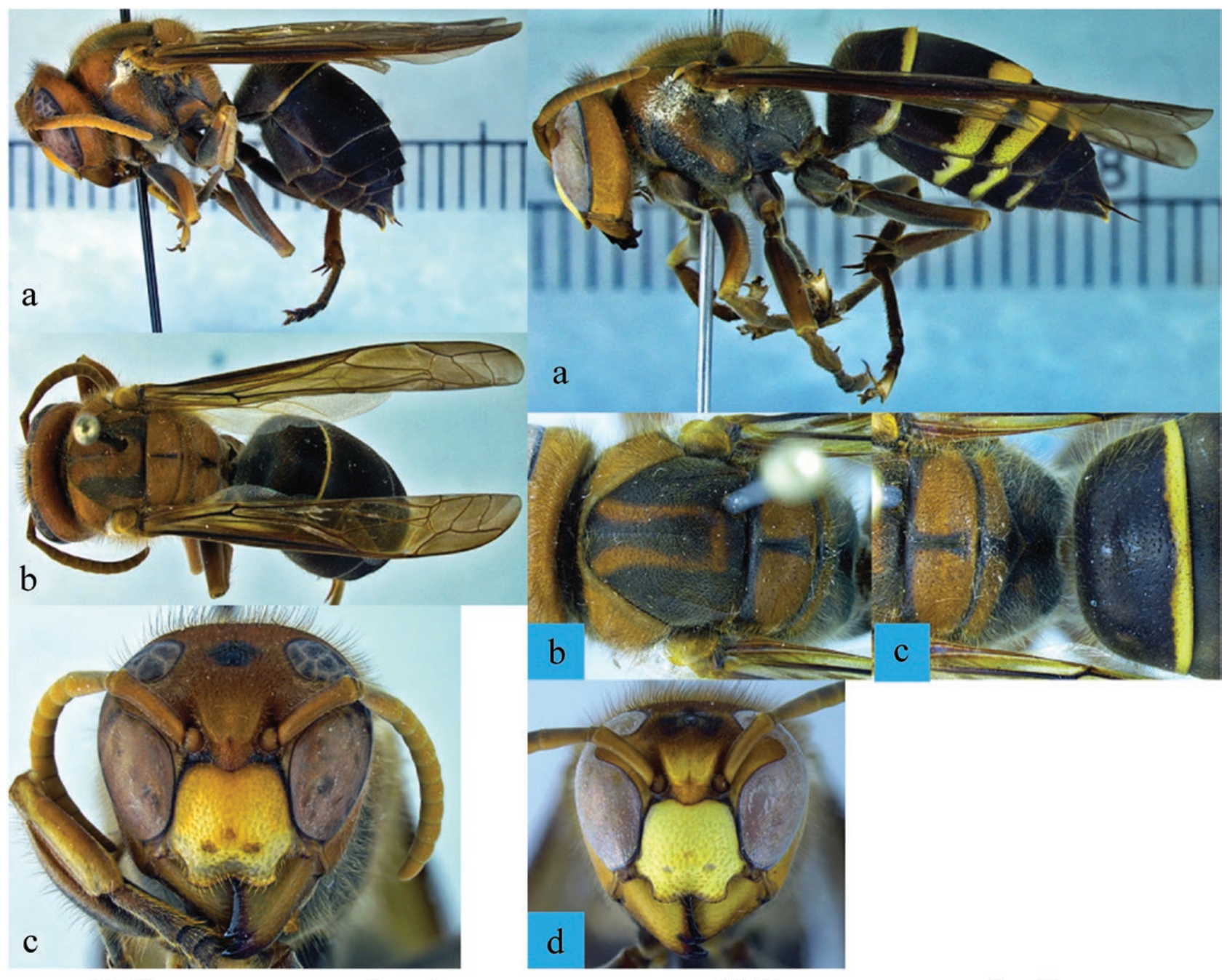

36. Vespa mocsaryana form A

\section{Vespa mocsaryana form $\mathrm{B}$}

Figs. 36 and 37. Vespa mocsaryana color forms. (a) Lateral view. (b) Dorsal view. (c) Front view of face.

Vespa nilotica Vallot, 1802: 170; Type unknown; Egypt (repository unknown).

Vespa jurinei de Saussure, 1854: 133; Lectotype female (designated by Guiglia, 1971: 94); Albania (LONDON-NHM).

Vespa orientalis var. aegyptiaca André, 1884: 584' Type unknown; Egypt: Cairo, Alexandria 'Le Caire, Alexandrie' (PARIS). Nec Vespa aegyptiaca Vallot, 1802.

Vespa indica Wroughton, 1889: 35. Lapsus for Vespa orientalis Linnaeus.

Vespa orientalis var. zavattarii Guiglia and Capra, 1933: 168. Syntype females; Libya: 'Fezzan, Ubari; Algeria: Oued Tizzi' (GENOA).

Vespa orientalis var. somalica Giordani Soika, 1934: 184. Lectotype female (designated by Giordani Soika, 1973: 11); Somalia: 'Somalia italiana di Carim' (VENICE).

Vespa orientalis arabica Giordani Soika, 1957: 482. Holotype female; Yemen: 'Western Aden Protectorate: Al Milah' (LONDON-NHM).

Distribution. north Africa, southern Europe, Turkey, Middle East, southern Russia, Afghanistan, Pakistan, India, Nepal, China (Xinjiang). Introduced into Czech Republic; Spain; Madagascar; Mexico.
Discussion. This is a distinctively marked reddish orange and yellow wasp. The nests are built in the ground in rodent burrows or rock crevices (Archer 1998a). Around human habitation, nests have also been found in empty beehives, under eaves or floorboards, and even in abandoned outhouses. It has been recorded from Mexico but is evidently not established (Dvořák 2006). Vespa orientalis are scavengers, feeding on nectar and fruit as well as insects and carrion. They also prey on honeybees and other pollinators. A study by Plotkin et al. (2010) found that this species has a novel form of photosynthesis using yellow pigments in the cuticle.

\section{Vespa philippinensis de Saussure}

Figs. $6 \mathrm{a}, 6 \mathrm{c}, 7 \mathrm{a}, 8 \mathrm{a}, 8 \mathrm{c}$, and 41

Vespa philippinensis de Saussure, 1854: 148. Holotype male; Philippines (LONDON-NHM).

Distribution. Philippines (Leyte, Luzon, Negros, Samar).

Discussion. Little is known about the biology of this endemic species. A nest described by Starr (1987) from the island of Leyte was found in a cavity in the ground (Archer 1991). 


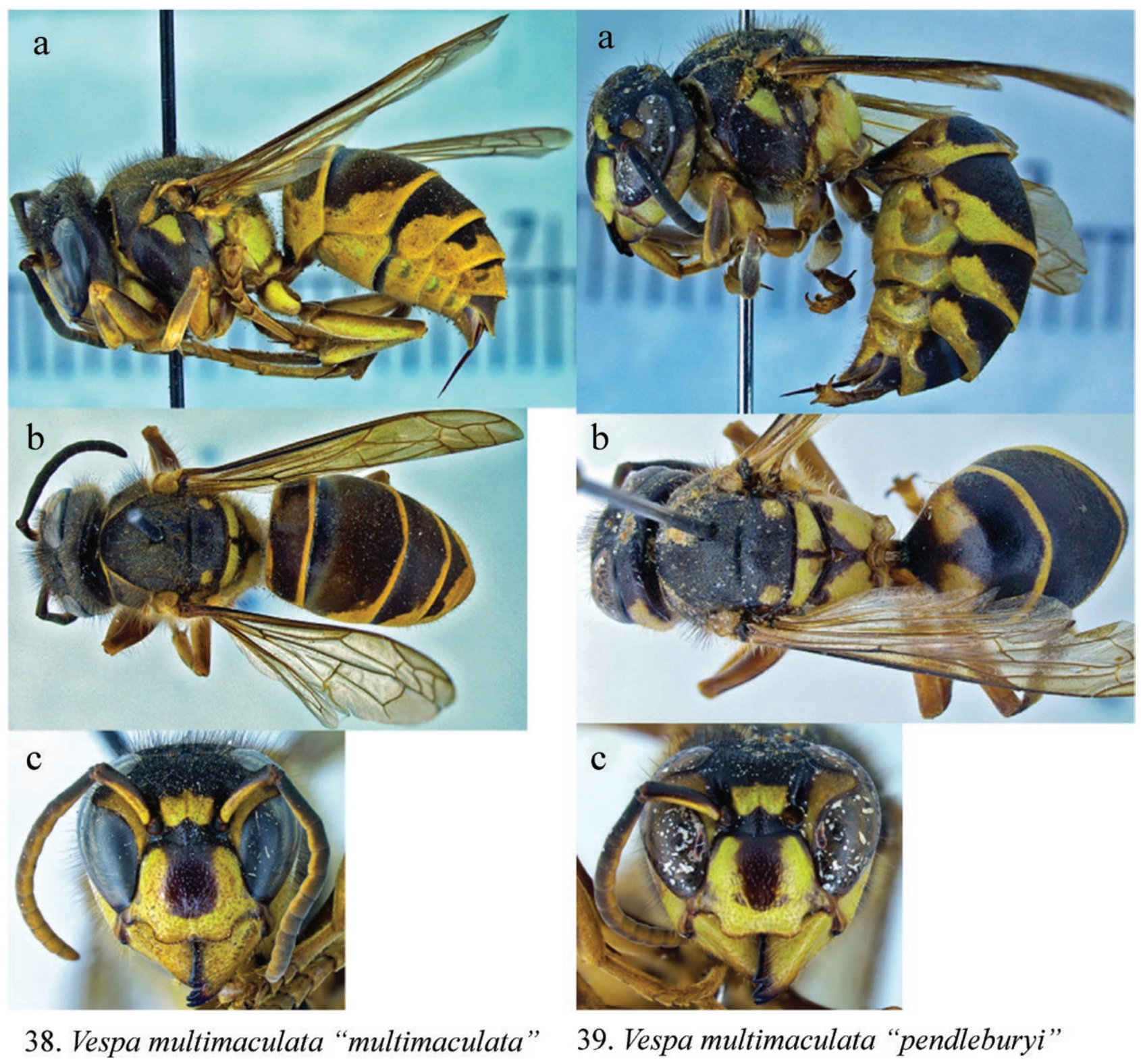

Figs. 38 and 39. Vespa multimaculata color forms. (a) Lateral view. (b) Dorsal view. (c) Front view of face.

\section{Vespa simillima Smith}

Figs. 42 and 43

Vespa simillima Smith, 1868: 280. Holotype female; Japan: 'Hakodadi' (LONDON-NHM).

Vespa mongolica André, 1884: 4. Syntype male, female; Russia: 'Vladivostock, sur l'Amour, dans la Sibérie orientale' (PARIS?).

Vespa xanthoptera Cameron, 1903: 278. Holotype male; Japan: 'Michzusawa' (LONDON-NHM).

Vespa micado Cameron, 1903: 279. Lectotype female (designated by Kojima, 1997: 20); Japan: Nagasaki (LONDON-NHM).

Vespa mongolica var. sexpunctata Pérez, 1905: 24. Holotype female; Japan: 'Yokohama' (PARIS).

Vespa mongolica var. flavata Pérez, 1910: 17. Holotype female; China (PARIS).

Distribution. China (Liaoning), southwestern Russia, Korea, Japan (including Yaku-shima Is.). Introduced into British Columbia, Canada, but not established; Taiwan?
Discussion. Nests of this species have been found in the ground, and in sheltered above ground sites, such as hollow trees, wall voids, and attics. A queen of $V$. simillima was found in the village of Tsaochan in Taiwan in 2003, but it is unclear whether the species has established on the island (Sung et al. 2006). It is apparently not established in British Colombia (Kimsey and Carpenter 2012).

\section{Vespa soror du Buysson}

Figs. 3a, 3c, and 44

Vespa ducalis var. soror du Buysson, 1905 (1904): 519. Lectotype female (designated by van der Vecht, 1957: 16); China: Jaingxi, 'Kiang-si' (PARIS). Given as mandarina soror by van der Vecht, 1957.

Distribution. India, southwestern China, Hong Kong, Thailand, Laos, Vietnam.

Discussion. Little biology is known for this species. It has been found in hilly and montane sites up to 1,500 $\mathrm{m}$ in continental southeastern 


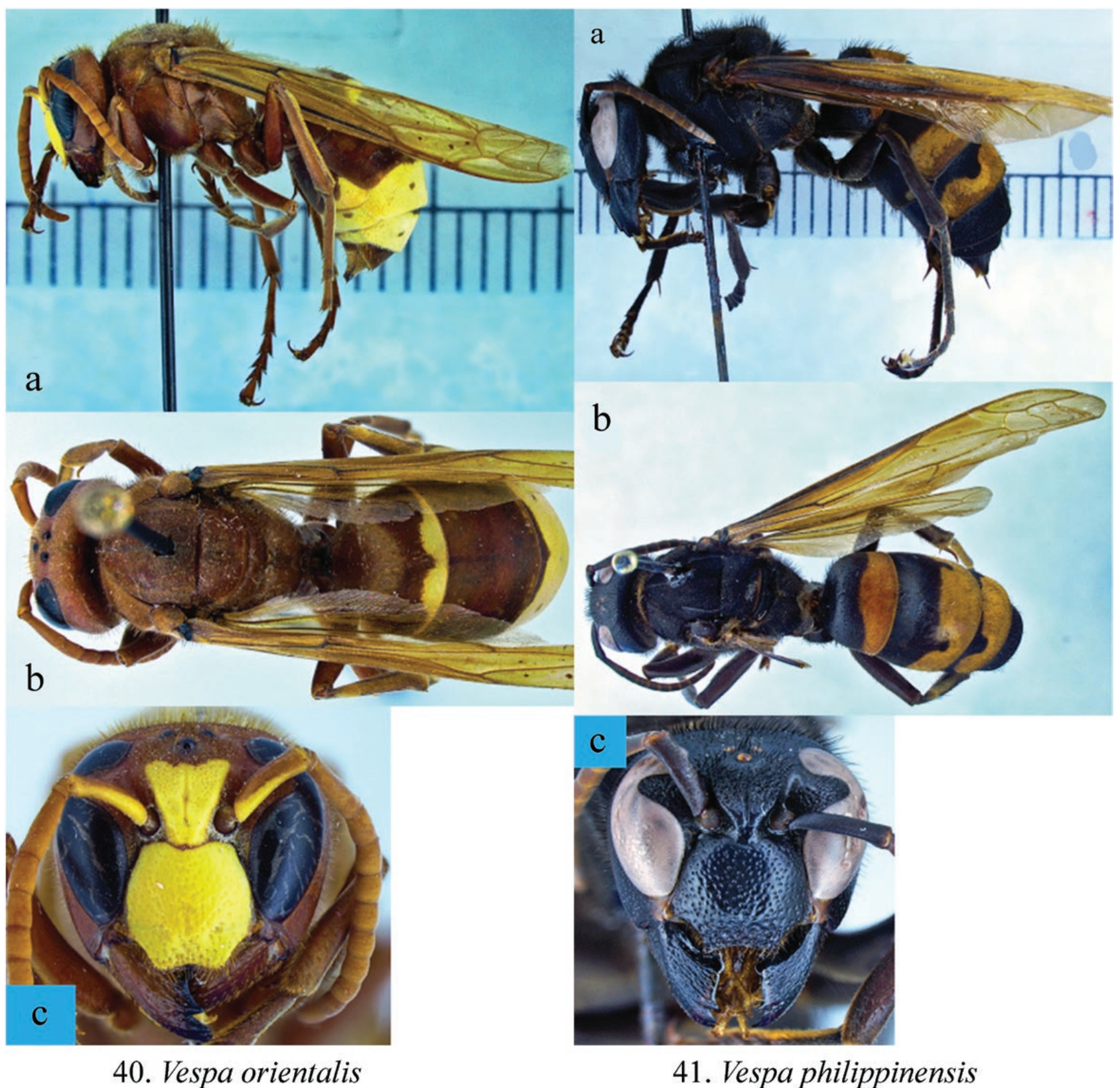

Figs. 40 and 41. Vespa color forms, (a) Lateral view. (b) Dorsal view. (c) Front view of face. Fig. 40. V. orientalis. Fig. 41. V. philippinensis.

Asia (Archer 2008). The nests are typically underground. These wasps are predators of other insects and even small vertebrates, like geckos. They will also attack nests of honeybees and other social vespids, including hornets (Lee 2009).

\section{Vespa tropica (Linnaeus)}

Figs. 7b, 7e, 7f, and 45-49

Sphex tropica Linnaeus, 1758: 571. Holotype female; 'in Indiis' (UPPSALA).

Vespa cincta Fabricius, 1775: 362. Holotype?; India: 'ad littora Malabarica' (LONDON-LS). Nec Vespa cincta Drury, 1773.

Vespa crabro tenebrionis Christ, 1791: 216. Type? (destroyed).

Vespa deusta Lepeletier, 1836: 506. Lectotype female (designated by van der Vecht, 1959: 226); 'Patrie inconnue' (TURIN).
Vespa unicolor Smith, 1863: 44. Holotype female; Indonesia: 'Bouru' (OXFORD).

Vespa cinta Wroughton, 1889: 35. Misspelling of Vespa cincta Degeer 1773.

Vespa eulemoides du Buysson, 1905 (1904): 530. Holotype female; India: 'Ile Andaman: port de Blair' (GENOA).

Vespa affinis var. trisignata Pérez, 1910: 8. Holotype female; Indonesia: 'Timor' (PARIS).

Vespa rubricans Pérez, 1910: 10. Lectotype female (designated by van der Vecht 1957); 'Lindi (Afrique orientale allemande)' (PARIS).

Vespa tropica var. anthracina Bequaert, 1936: 341. Holotype female; Philippines: 'Sibuwan' (WASHINGTON).

Vespa tropica var. haematodes Bequaert, 1936: 338. Holotype female; India: 'Kooloo' (CAMBRIDGE). 


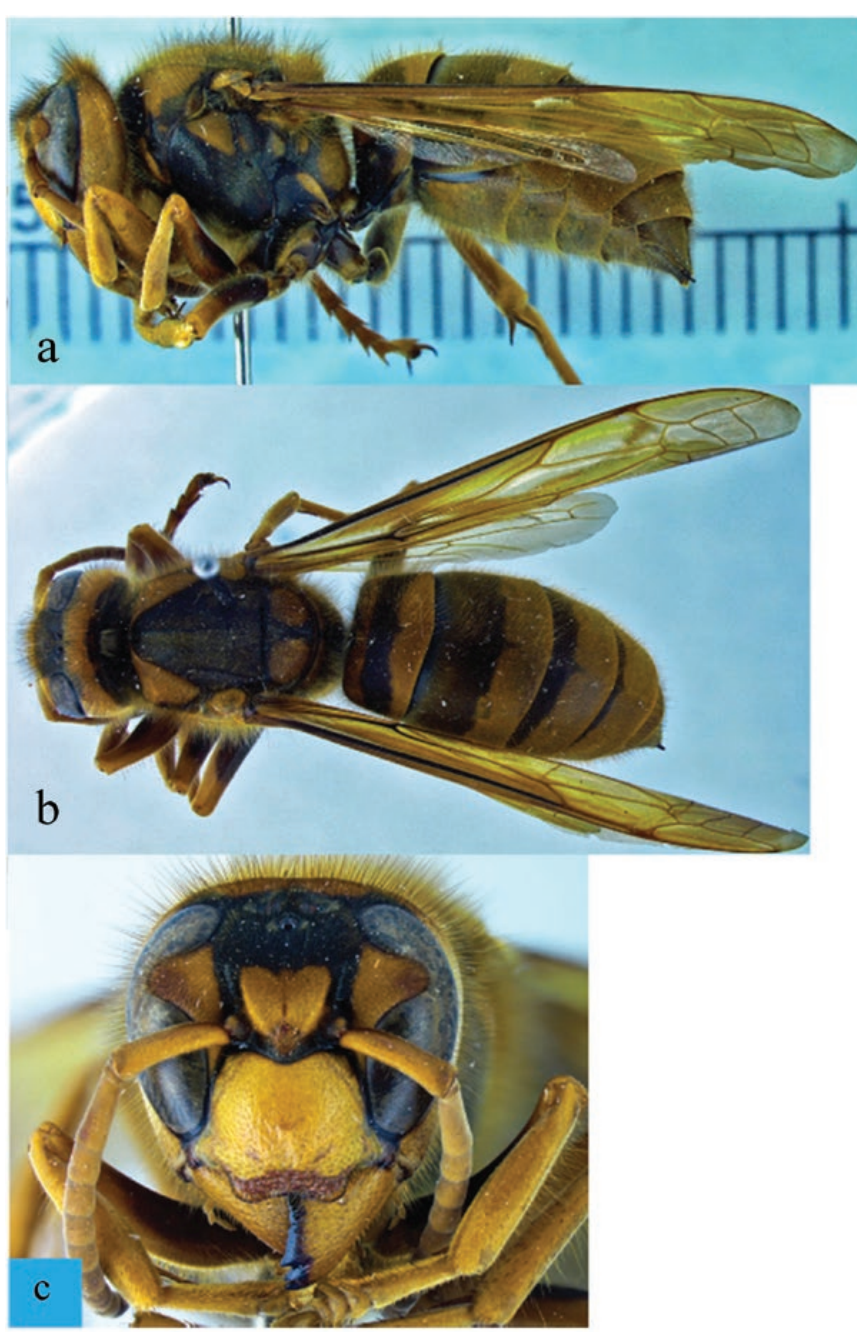

42. Vespa simillima "mongolica"

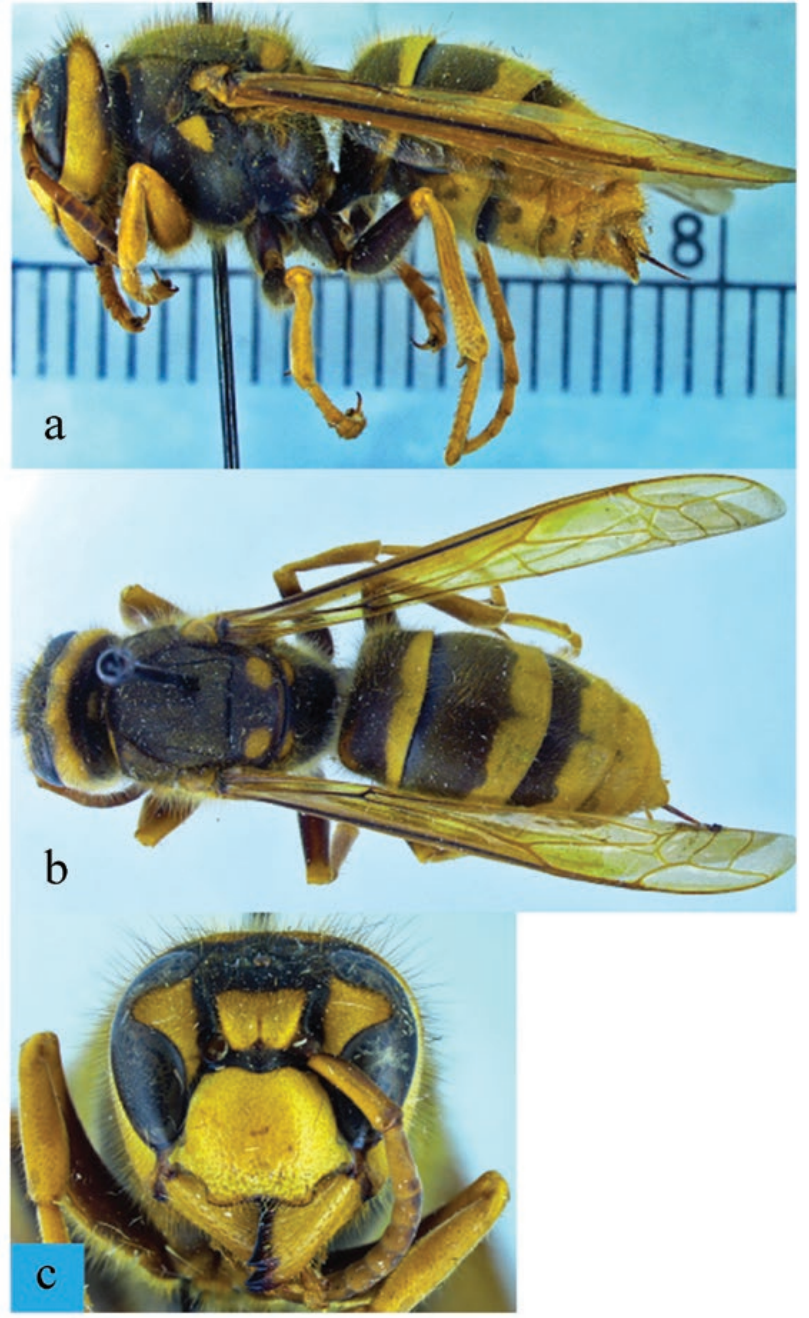

43. Vespa simillima "xanthoptera"

Figs. 42 and 43. Vespa simillima color forms. (a) Lateral view. (b) Dorsal view. (c) Front view of face.

Vespa tropica leefmansi van der Vecht, 1957: 19. Holotype female; Indonesia: 'Solok, Padang, Sumatra' (LEIDEN)

Vespa tropica trimeres van der Vecht, 1957: 19; Holotype female; Indonesia: Sulawesi, 'Palu, West Celebes' (LEIDEN).

Vespa tropica cebuana Kojima and Reyes, 1984: 260. Holotype female; Philippines: 'Cebu City' (TOKYO).

Distribution. Afghanistan, Pakistan, India, Sri Lanka, Bhutan, Nepal, southeastern China, Hong Kong, Myanmar, Thailand, Laos, Cambodia, Vietnam, Malaysia, Malaya, Borneo, Indonesia, Papua New Guinea (including New Britain), Philippines; exotic in Guam. Discussion. This is a distinctively colored Vespa, with a black metasoma, except for a broad yellow to orange stripe across most of the second segment (as in $V$. affinis), although some individuals may lack this stripe. Vespa tropica are nest predators of other social wasps as well as other large-bodied insects, including honeybees (Archer 1991). Nests are either above ground in tree cavities or in cavities in the ground. Around human habitations, they will build nests under eaves, in shed, and attics.

\section{Vespa velutina Lepeletier}

Figs. 6b, 6d, 13d, and 50-52

Vespa velutina 'De Haan' Lepeletier, 1836: 507. Holotype female; Indonesia: 'Java' (TURIN?).

Vespa auraria Smith, 1852: 46. Syntype females; 'Northern India' (LONDON-NHM, OXFORD).

Vespa crabro var. immaculata Morawitz, 1889: 161. Holotype female; China: 'Kansu. Nanpin'. (ST. PETERSBURG). Nec Vespa immaculata Gmelin, 1790.

Vespa frubstorferi Stadelmann, 1894: 89. Holotype female; Indonesia: West-Java, Gunung Gede, 'in einer Höhe, 8000 ft'. (Berlin?).

Vespa velutina var. ardens du Buysson, 1905 (1904): 550. Syntype female, male; Indonesia: Lombok, Sapit, Sumbawa Islands. (Vienna, Budapest).

Vespa auraria var. nigrithorax du Buysson, 1905 (1904): 553. Lectotype female (designated by van der Vecht, 1957: 37), India: Darjiling (PARIS). 


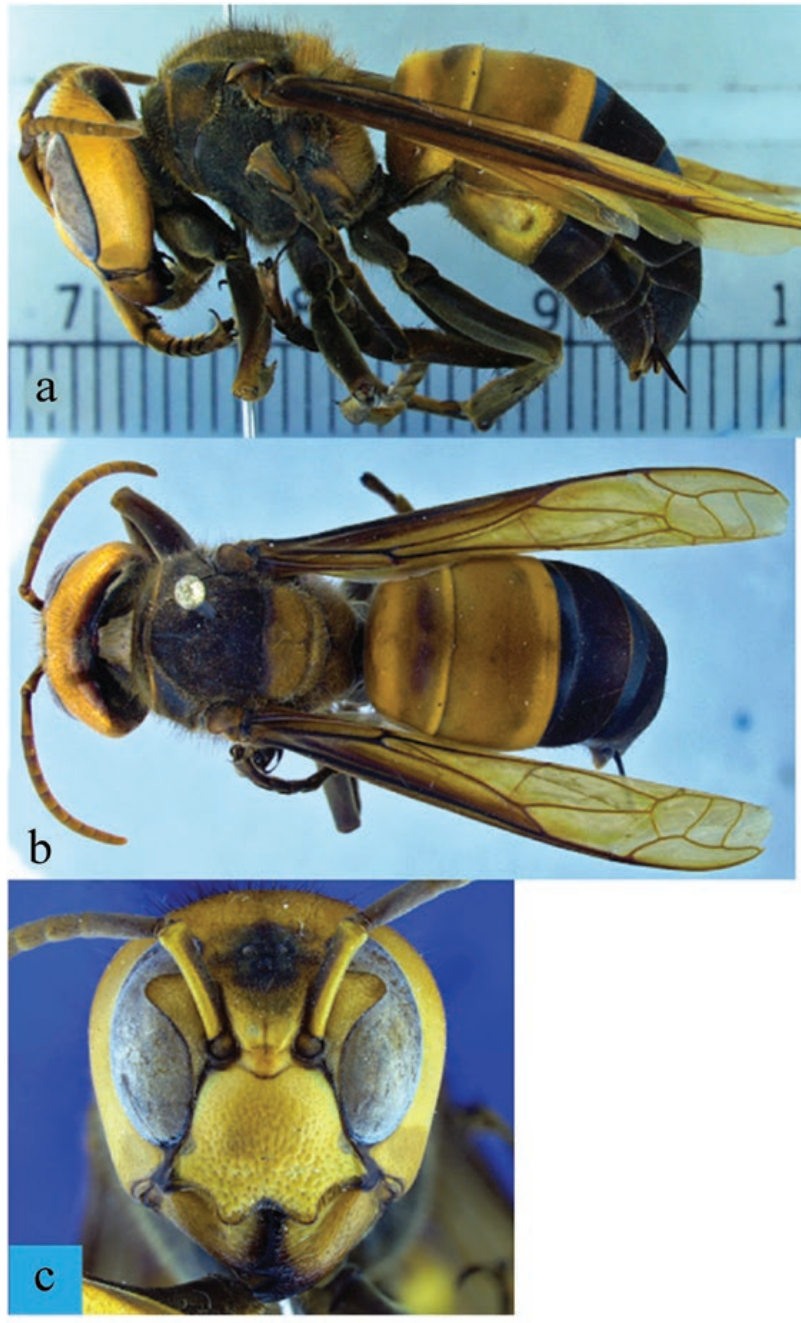

44. Vespa soror a

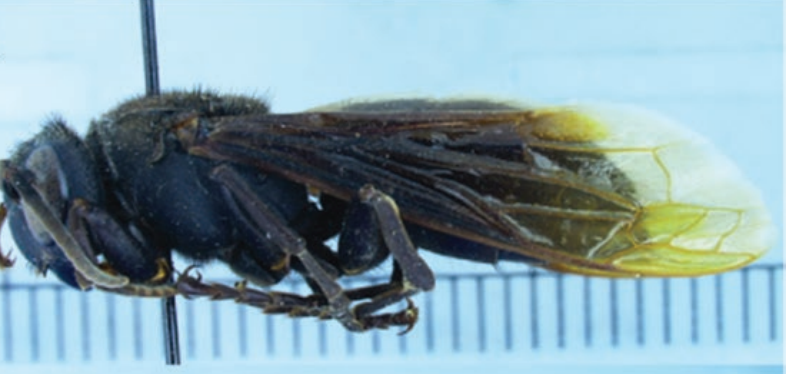

$\mathrm{b}$

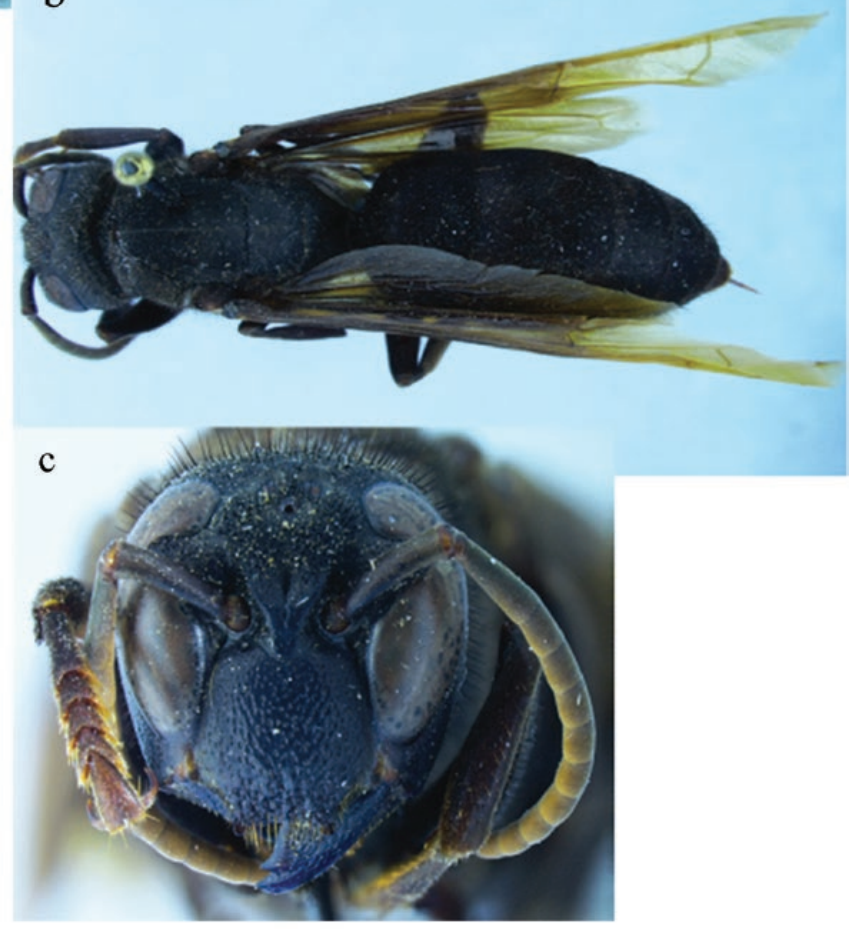

45. Vespa tropica

Figs. 44 and 45. Vespa color forms. (a) Lateral view. (b) Dorsal view. (c) Front view of face. Fig. 44. V. soror. Fig. 45. V. tropica.

Vespa velutina var. celebensis Pérez, 1910: 12. Holotype female; Indonesia: Sulawesi, 'Bua-Kraëng' (PARIS).

Vespa velutina var. megei Pérez, 1910: 13. Holotype female; China: 'Koueï-Tchéou (Chine centrale)' (PARIS). Synonymized by van der Vecht, 1957: 37.

Vespa mongolica var. divergens Pérez, 1910: 16. Holotype female; Malaysia: Perak (PARIS).

Vespa flavitarsus Sonan, 1929: 142. Holotype female; Taiwan: 'Taiko-san (Kagi)' (coll. M. Kato).

Vespa auraria flavitarsis Ma, 1937: 31. Misspelling of Vespa flavitarsus Sonan, 1929?

Vespa velutina variana van der Vecht, 1957: 37. Holotype female: Thailand: Ban Umphang, Doi Hua Mot (LEIDEN).

Vespa velutina karnyi van der Vecht, 1957: 38. Holotype female; Indonesia: Sumatra: Bandar Buru, Deli (LEIDEN).

Vespa velutina sumbana van der Vecht, 1957: 40. Holotype female; Indonesia: Sumba Is., Kananggar (LEIDEN).

Vespa velutina timorensis van der Vecht, 1957: 40. Holotype female; Indonesia: Timor Is., Mollo (BASEL).
Vespa velutina floresiana van der Vecht, 1957: 40. Holotype female; Indonesia: Flores Is., Labuan Badjo (LEIDEN).

Vespa velutina pruthii van der Vecht, 1959: 228. Holotype female; India: Kashmir, Srinagar (CALCUTTA).

Distribution. Afghanistan, Pakistan, India, Bhutan, Nepal, southern China, Hong Kong, Taiwan, Myanmar, Thailand, Laos, Vietnam, Malaysia, Malaya, Indonesia. Introduced into Korea, Japan (Tsushima I.), France, Belgium, United Kingdom, Spain, Portugal, Italy, Yemen.

Discussion. Vespa velutina has distinctive yellow tarsi and velvety appearing dark brown to black thorax and metasoma. It is native across Asia. However, V. velutina was introduced into Europe apparently with Chinese pottery in the past couple of decades (Keeling et al. 2017). Although this species preys on a variety of insects, including flies, dragonflies, and Orthoptera, it is a notorious predator of pollinators, particularly honeybees (Apis mellifera and A. cerana). Apis cerana have adapted to this hornet 


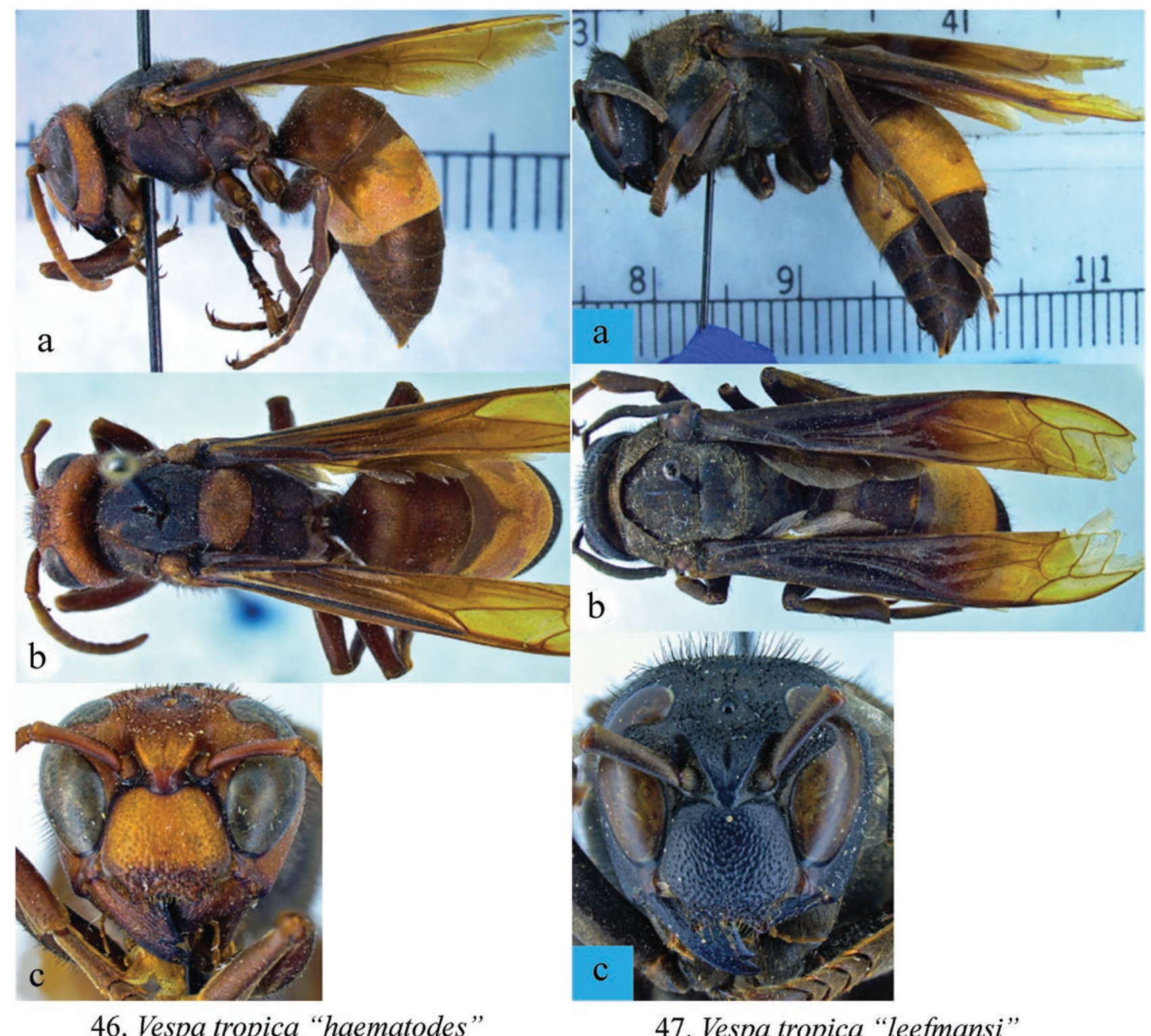

Figs. 46 and 47. Vespa tropica color forms. (a) Lateral view. (b) Dorsal view. (c) Front view of face.

using avoidance behaviors and balling behaviors where they heat the hornet to death (Abrol 2006).

\section{Vespa vivax Smith}

Figs. $13 \mathrm{~d}$ and 53

Vespa vivax Smith (in Horne and Smith), 1870: 190. Holotype female; India: Binsar, 'Binsur, Kumaon' (LONDON-NHM).

Vespa velutina var. mediozonalis Pérez, 1910: 14. Holotype female; India: Sikkim (PARIS).

Vespa wilemani Meade-Waldo, 1911: 104. Holotype female; Taiwan: 'Formosa' (LONDON-NHM).

Distribution. India, Nepal, Myanmar, Thailand, China (Sichuan, Yunnan, Tibet), Taiwan.

Discussion. Little is known about this south Asian Vespa species. In Taiwan, nests have been found in montane regions at elevations between 1,500 and 2,500 $\mathrm{m}$ (Archer 1994).

\section{Conclusion}

From 2010 to 2018, there have been close to 50 interceptions of Vespa (hornets) and Vespula (yellow jackets (Vespula) at US ports of entry. Little less than half of those interceptions were hornets. The Vespa species intercepted include V. bellicosa, V. crabro, V. orientalis, $V$. mandarinia, and V. tropica. One of the interceptions of significance was an entire nest of V. mandarinia containing live brood and pupae that was sent via express courier from Asia.

All species of Vespa, except V. crabro, which is already introduced into the eastern United States, are considered of quarantine importance by the USDA-APHIS. As part of the work of monitoring for possible introductions of hornets in the United States, one of us (AHSP) created a website, Invasive Hornets (https://www. ipmimages.org/browse/projectthumb.cfm? proj=1159), which is part of a cooperation between the USDA, Animal Plant Health Inspection Service (APHIS), Plant Protection and Quarantine (PPQ) and the University of Georgia. This website contains more than 1,000 


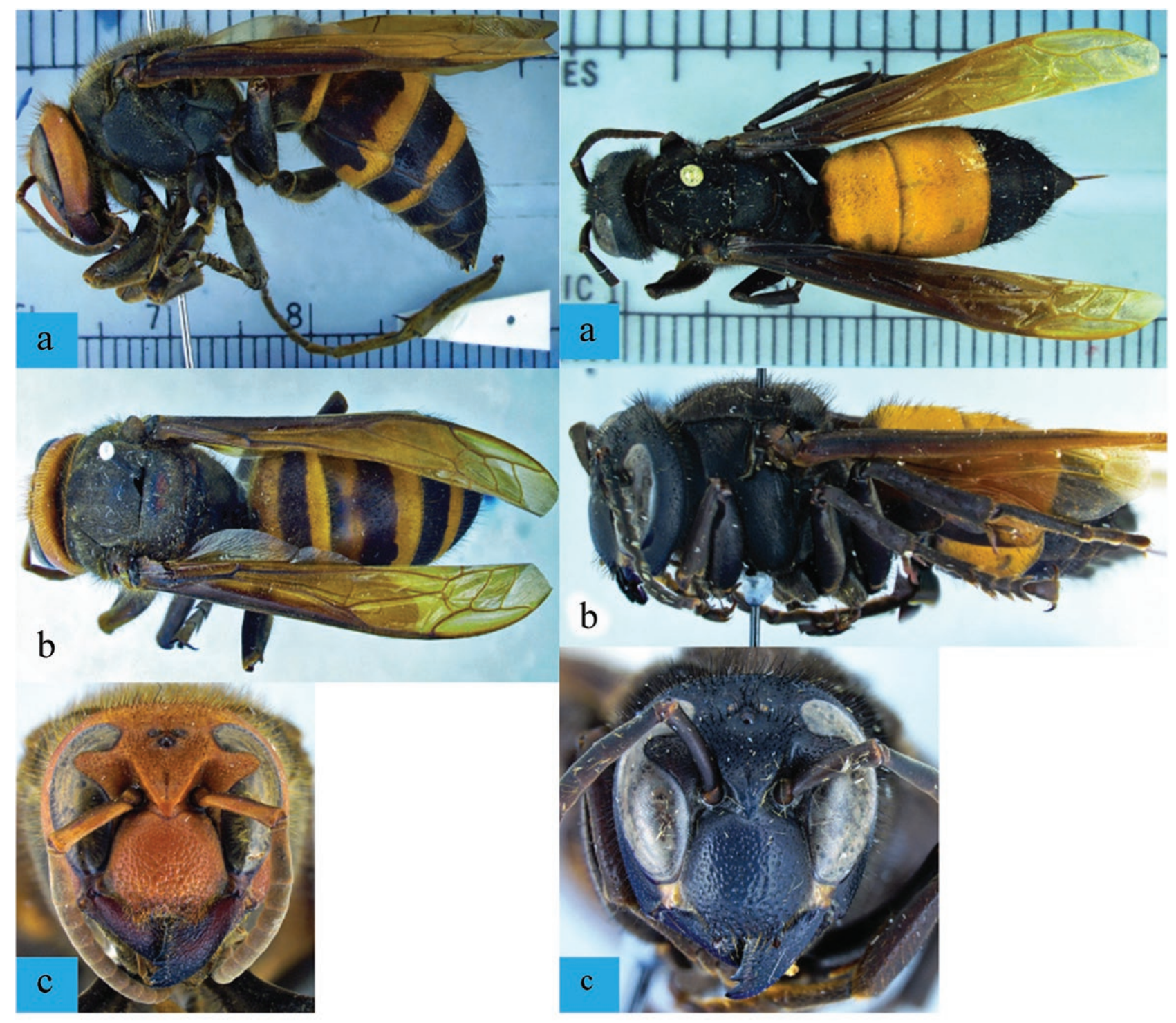

48. Vespa tropica "pulchra"

49. Vespa tropica "trimeres"

Figs. 48 and 49. Vespa tropica color forms. (a) Lateral view. (b) Dorsal view. (c) Front view of face. 


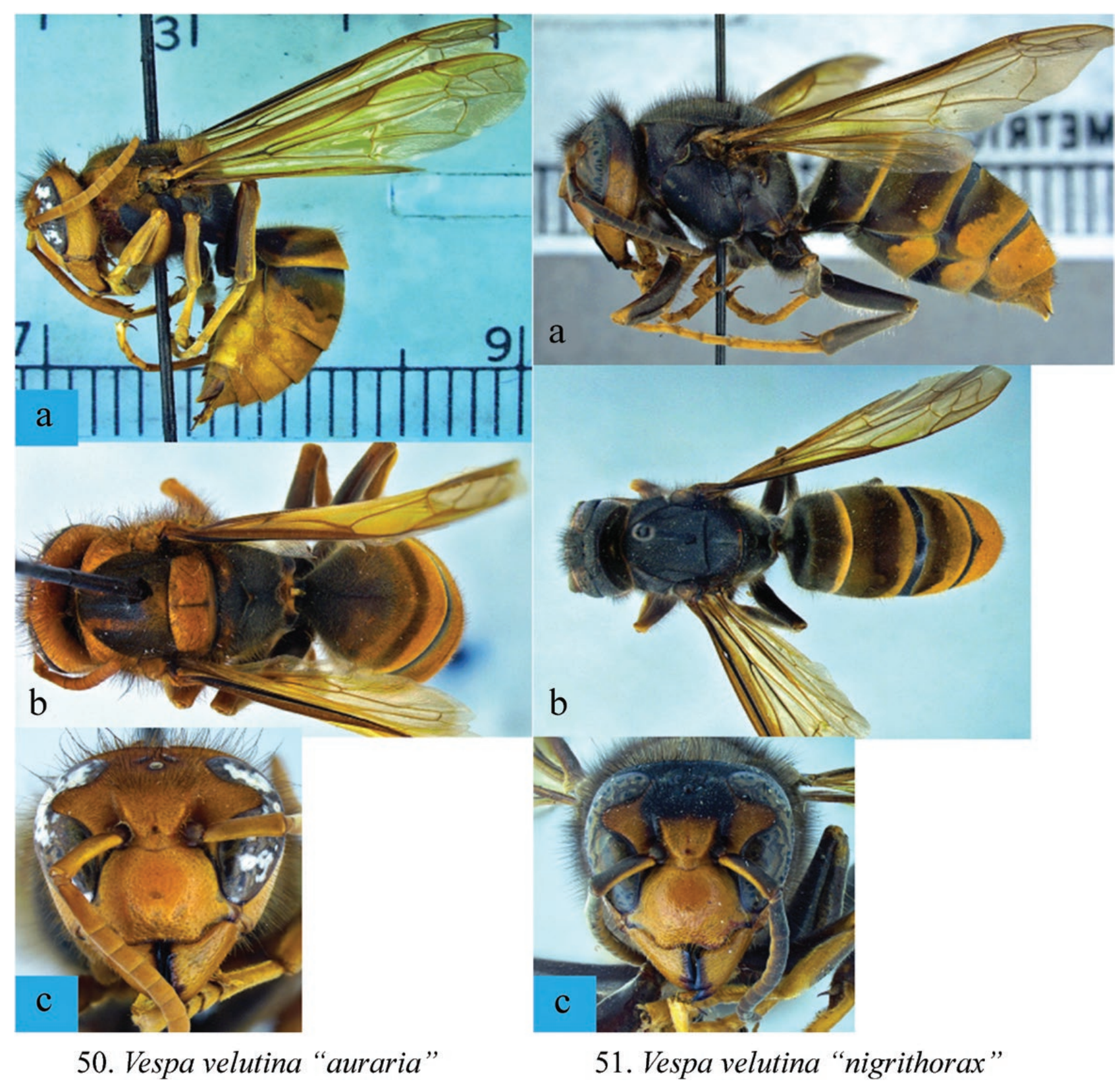

Figs. $\mathbf{5 0}$ and 51. Vespa velutina color forms. (a) Lateral view. (b) Dorsal view. (c) Front view of face. 


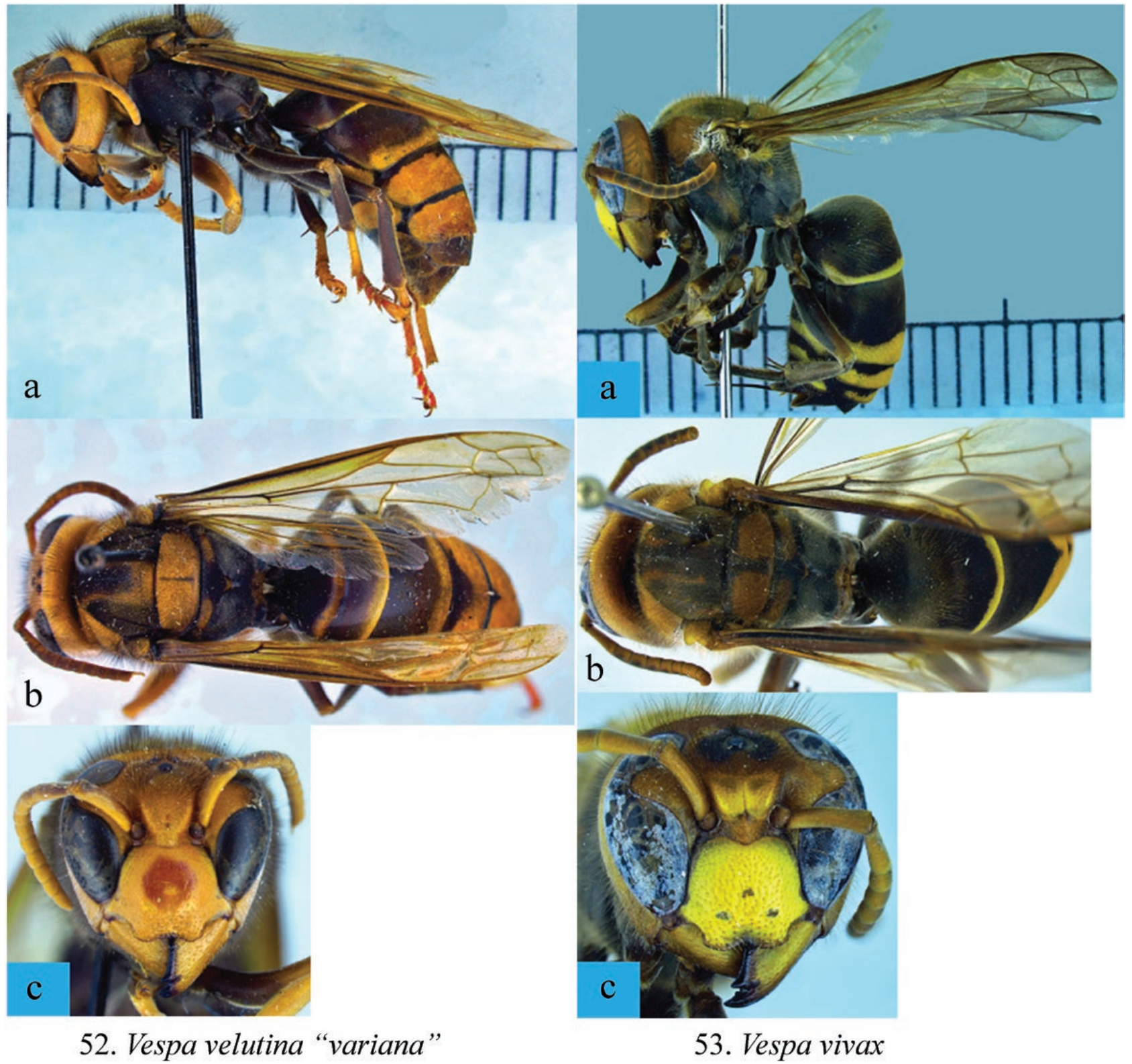

Figs. 52 and 53. Vespa color forms, (a) Lateral view. (b) Dorsal view. (c) Front view of face. Fig. 53. V. velutina. Fig. 54. V. vivax.

stacked, high-quality images of all the species and most of the races of the genus Vespa.

It is important to have the resources for the identification and prevention of introduction of non-native species and to understand the potential effects of invasive hornets in our ecosystems. Hornets are dangerous for the beekeeping industry because they can alter pollination in agriculture and disrupt the beekeeping industry, as well as create public health and safety problems.

\section{Acknowledgments}

We want to thank Christine Lebeau and Steve Heydon for helping to process the loan of Vespa material from the AMNH and the BME, respectively. We would also like to thank Mary Burns from National Identification Services (NIS) of the USDA-APHIS- Plant Protection and Quarantine (PPQ) for providing information about the number of interceptions of Vespa at U.S. ports of entry. AHS-P wants to thank USDA-APHIS-PPQ Field Operations for allowing him the time to obtain and process the im- ages in this work and to PPQ- Science and Technology for supporting his work with bees and hornets. This is a contribution of the United States Department of Agriculture, Animal Plant and Health Inspection Service, Plant Protection and Quarantine- Science and Technology, Identification Technology Program (ITP).

\section{References Cited}

Anonymous. 2016. The greater banded hornet has found its way to Guam. University of Guam Research and Extension. Available from https:// cnas-re.uog.edu/the-greater-banded-hornet-has-found-its-way-to-guam/.

Anonymous. 2020. Three Asian giant hornets found in Nanaimo. Available from https://bcinvasives.ca/news-events/news-from-iscbc/bc-governmentbulletin-three-asian-giant-hornets-found-in-nanaimo

Abrol, D. P. 1994. Ecology, behaviour and management of social wasp, Vespa velutina Smith (Hymenoptera: Vespidae), attacking honeybee colonies. Korean J. Apicul. 9: 5-10.

Abrol, D. P. 2006. Defensive behaviour of Apis cerana F. against predatory wasps. J. Apicul. Sci. 50: 39-46. 
Archer, M. E. 1989. A key to the world species of the Vespinae (Hymenoptera). Part 1 Keys, Checklist and Distribution. Part 2 Figures. Research Monograph of the College of Ripon and York, St. John, UK. 2: 1-47.

Archer, M. E. 1991. Taxonomy and bionomics of the Vespa tropica group. Entomol. Mon. Mag. 127: 225-232.

Archer, M. E. 1993. The life history and colonial characteristics of the hornet, Vespa crabro L. Entomol. Mon. Mag. 129: 151-163.

Archer, M. E. 1994. Taxonomy, distribution and nesting biology of Vespa bicolor group. Entomol. Monthly Mag. 130: 149-158.

Archer, M. E. 1995. Taxonomy, distribution and nesting biology of the Vespa mandarinia group. Entomol. Mon. Mag. 131: 47-53.

Archer, M. E. 1997. Taxonomy, distribution and nesting biology of Vespa affinis (L.) and Vespa mocsaryana du Buysson. Entomol. Mon. Mag. 133: 27-38.

Archer, M. E. 1998a. Taxonomy, distribution and nesting biology of Vespa orientalis L. Entomol. Mon. Mag. 134: 45-51.

Archer, M. E. 1998b. Taxonomy, distribution and nesting biology of Vespa analis F. Entomol. Mon. Mag. 134: 215-222.

Archer, M. E. 1999. Taxonomy, distribution and nesting biology of Vespa binghami, V. basalis, V. variabilis, V. fervida, V. luctuosa, V. multimaculata and V. bellicosa. Entomol. Monthly Mag. 135: 43-50.

Archer, M. E. 2008. Taxonomy, distribution and nesting biology of species of the genera Provespa Ashmead and Vespa Linnaeus (Hymenoptera, Vespidae). Entomologist's Monthly Magazine. 144: 69-101.

Archer, M. E. 2011. Analysis of a nest of Vespa multimaculata Perez, 1910. Malay. Nat. J. 63: 561-567.

Archer, M. E. 2012. Vespine wasps of the World: behaviour, ecology \& taxonomy of the vespinae (Monograph Series). Siri Scientific Press, U.K. 352p.

Baracchi, D., G. Cusseau, D. Pradella, and S. Turillazzi. 2010. Defense reactions of Apis mellifera ligustica against attacks from the European hornet Vespa crabro. Ethol. Ecol. \& Evol. 22: 1-14.

Beggs, J. R., G. E. G. Brockerhoff, J. C. Corley, M. Kenis, M. Masciocchi, F. Muller, Q. Rome and C. Villemant. 2011. Ecological effects and management of invasive alien Vespidae. BioControl 56: 505-526.

Bequaert, J. 1932. A tentative synopsis of the hornets and yellow-jackets (Vespinae; Hymenoptera) of America. Entomol. Am. (n. s.) 12: 71-138.

Blanchard, P, F. Schurr, O. Celle, N. Cougoule, P. Drajnudel, R. Thiéry, J.P. Faucon and M. Ribiére. 2008. First detection of Israeli acute paralysis virus (IAPV) in France, a dicistrovirus affecting honeybees (Apis mellifera). J. Invert. Pathol. 99: 348-350.

Brodman, J., R. Twele, F. Wittko, Y.-b. Luo, S. Xi-qiang, and M. Ayasse. 2009. Orchid mimics honey bee alarm pheromone in order to attract hornets for pollination. Current Biol. 19: 1368-1372.

Carpenter, J. M., and J. Kojima. 1997. Checklist of the species in the subfamily Vespinae. Nat. Hist. Bull., Ibaraki Univ. 1: 51-92.

Carpenter, J. M., L. Dvorak, J.-L. Kojima, L.T.P. Nguyen, A. Perrard, and K. M. Pickett. et al. 2011. Taxonomic notes on the Vespinae of Yunnan. Amer. Mus. Nov. (3709): 1-10.

Choi M. B., S. J. Martin, and J. W. Lee. 2011. Distribution, spread, and impact of the invasive hornet Vespa velutina in South Korea. J. Asia-Pacific Entomol. 15: 473-477.

Cini, A., F. Cappa, I. Petrocelli, I. Pepiciello, L. Bortolotti, R. Cervo. 2018. Competition between the native and the introduced hornets Vespa crabro and Vespa velutina: a comparison of potentially relevant life-history traits. Ecol. Entomol. 43: 351-362.

Cox-Foster, D. L., S. Conlan, E. C. Holmes, G. Palacios, J. D. Evans, N. A. Moran, P.-L. Quan, T. Briese, M. Hornig, and D. M. Geiser. 2007. A metagenomic survey of microbes in honeybee colony collapse disorder. Science 318: 283-287.

Dvořák, L. 2006. Oriental Hornet Vespa orientalis Linnaeus, 1771 found in Mexico (Hymenoptera, Vespidae, Vespinae). Entomological Problems 36: 80.
Fedele, E., E. Gervasini, A. C. Cardoso, A. La Notte, S. Vallecillo, K. Tsiamis, and J. Maes. 2019. Invasive Alien species impact on ecosystem services - Asian hornet (Vespa velutina nigrithorax) case study. EUR 29827 EN, Publications Office of the European Union, ISBN 97892-76-09511-8, doi:10.2760/134398, JRC 111718.

Keeling, M. J., D. N. Franklin, S. Datta, M. A. Brown, and G. E. Budge. 2017. Predicting the spread of the Asian hornet (Vespa velutina) following its incursion into Great Britain. Sci. Rep. 7: 1-7. doi:10.1038/ s41598-017-06212-0.

Kimsey, L. S., and J. M. Carpenter. 2012. The Vespinae of North America (Vespidae, Hymenoptera). J. Hym. Res. 28: 37-65.

Kularatne, K., T. Kannangare, A. Jayasena, R. Waduge, K. Weerakoon, and S. A. Kularatne. 2014. Fatal acute pulmonary oedema and acute renal failure following multiple wasp/hornet (Vespa affinis) stings in Sri Lanka: two case reports. J. Med. Case Rep. 8: 188.

Landolt P. J., J. M. Siera, T. R. Unruh, and R. S. Zack. 2010. A new species of Vespula, and first record of Vespa crabro L. from Guatemala, Central America. Zootaxa 2629: 61-68.

Lee, J. X. 2009. A note on Vespa soror in Hong Kong. Hong Kong Entomol. Bull. 1: 18-22.

Martin, S. J. 1995. Hornets (Hymenoptera: Vespidae) of Malaysia. Malay. Nat. J. 49: 71-78.

Martin, S. J., J. Takahashi, M. Ono, and F. P. Drijhout. 2008. Is the social parasite Vespa dybowskii using chemical transparency to get her eggs accepted? J. Ins. Physiol. 54: 700-707.

Matsura, M., and S. Yamane. 1990. Biology of the Vespinae wasps. SpringerVerlag, Berlin, Germany. 323p.

Monceau, K., O. Bonnard, and D. Thiéry. 2014. Vespa velutina: a new invasive predator of honeybees in Europe. J. Pest Sci. 87: 1-16.

Nguyen, B.H., H. L. Mai, T.X. Nguyen, et al. 2010. Swarming hornet attacks: shock and acute kidney injury-a large case series from Vietnam. Nephrol. Dial. Transplant 25: 1146-1150.

Nguyen, L. T. P., F. Saito, J.-I. Kojima, and J. M. Carpenter. 2006. Vespidae of Viet Nam (Insecta: Hymenoptera). 2. Taxonomic notes on Vespinae. Zool. Sci. 23: 95-104.

Perrard, A., M. Arca, Q. Rome, et al. 2014. Geographic variation of melanisation patterns in a hornet species: genetic differences, climatic pressures or aposematic constraints? PLoS One 9: e94162.

Plotkin, M., I. Hod, A. Zaban, et al. 2010. Solar energy harvesting in the epicuticle of the oriental hornet (Vespa orientalis). Naturwissenschaften 97: 1067-1076.

Saussure, H. de. 1898. Letter concerning insects introduced into the United States. Entomol. News 9: 144-145.

Shah F.A., and T. A. Shah. 1991. Vespa velutina, a serious pest of honey bees in Kashmir. BeeWorld 72: 161-164.

Shaw F. R., and J. Weidhaas Jr. 1956. Distribution and habits of the giant hornet in North America. J. Econ. Entomol. 49: 275.

Starr, C. K. 1987. A colony of the hornet Vespa philippinensis. Insectes Soc. 34: 109.

Sung, I.-H., S. Yamane, and K.-K. Ho. 2006. A new record of a hornet from Taiwan. Formosan Entomol. 26: 303-306.

Villemant, C., J. Haxaire, and J.-C. Streito. 2006a. Premier bilan de l'invasion de Vespa velutina Lepeletier en France (Hymenoptera, Vespidae). Bull. Soc. Entomol. France 111: 535-538.

Villemant, C., J. Haxaire, and J.-C. Streito. 2006b. The discovery of the Asian hornet Vespa velutina in France. Insectes 143: 3-7.

Yañez, O., H.-Q. Zheng, F.-L. Hu, P. Neuman, and V. Dietemann. 2012. A scientific note on Israeli acute paralysis virus infection of Eastern honeybee Apis cerana and vespine predator Vespa velutina. Apidologie 43: 587-589.

Zhang, J. F. 1989. Fossil insects from Shanwang, Shandong, China. Shandong Science and Technology, Shandong, China, Pub. 551 pp. 